\title{
Wie Mediennutzer in die Welt schauen: Die Newsrepertoires der Schweizerinnen und Schweizer und ihre Themenagenden
}

\author{
Schneider, Jörg ; Eisenegger, Mark
}

\begin{abstract}
Die Frage, wie Mediennutzer in die Welt schauen bzw. welches Bild sie von der Welt haben, untersuchen wir anhand einer Langzeitstudie, die seit 2009 einmal jährlich die Mediennutzung der Bevölkerung in der Schweiz untersucht und die Mediennutzung mit der Agenda der intensiv verfolgten Themen verknüpft. Aus den Daten zur Mediennutzung von inzwischen über 27000 Befragten werden sogenannte Newsrepertoires gebildet. Newsrepertoires erfassen die Kombination und die Nutzungsintensität der verschiedenen Medienangebote, die von einer einzelnen Person zu Informationszwecken konsumiert werden. Aus den individuellen Newsrepertoires lassen sich sechs Repertoiretypen aggregieren und soziodemografisch verorten. Stark in der traditionellen Medienwelt verhaftet sind die Repertoiretypen «Homeland Oriented», «Old World Boulevard» und «Old World Online», wobei der letztgenannte Typ die grösste Öffnung hin zur neuen, digitalisierten Medienwelt aufweist. In beiden Welten ist der Repertoiretyp der «Intensivnutzer» verankert, der sowohl traditionelle als auch online vermittelte Newsangebote ausgiebig in Anspruch nimmt. Am stärksten in der neuen Medienwelt beheimatet sind die Repertoiretypen der «Global Surfer» und «News Deprivierten». Es zeigt sich, dass diese beiden Typen in den letzten Jahren einen massiven Zuwachs verzeichnen und inzwischen die Mehrheit der Bevölkerung repräsentieren. Das ist deshalb bedenklich, weil in diesen beiden Newsrepertoires die konsumierte Medienqualität abfällt, d.h. eine unterdurchschnittliche Qualitätsbilanz zu konstatieren ist. Die «News Deprivierten» sind heute sogar die grösste Nutzungsgruppe überhaupt. Es handelt sich dabei um Nutzerinnen und Nutzer, die professionelle Newsangebote weit unterdurchschnittlich oft nutzen, und wenn, dann vor allem solche von minderer Qualität. Vor allem junge Frauen unter 30 Jahren sind in diesem Newsrepertoire überdurchschnittlich oft vertreten. Die Effekte der unterschiedlichen Newsrepertoires und der konsumierten Newsqualität zeigen sich in den Themenagenden, die die Repertoiretypen stark beachten. Die «Global Surfer» bevorzugen eine international ausgerichtete Themenagenda, aus der schweizerischen Medienarena ziehen sie sich zurück. Die «News-Deprivierten» nehmen vor allem Einzelereignisse und Bedrohungsszenarien wahr. Den Gegenpol zu diesem Repertoiretyp bilden die «Intensivnutzer», die aufgrund ihrer breiten Nutzung von qualitativ hochwertigen Informationsmedien eine vielfältige Themenagenda aufweisen und insbesondere auch Themenzusammenhänge wahrnehmen.
\end{abstract}

Posted at the Zurich Open Repository and Archive, University of Zurich

ZORA URL: https://doi.org/10.5167/uzh-130942

Scientific Publication in Electronic Form

Published Version

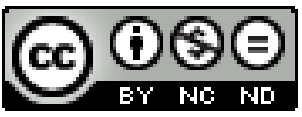

The following work is licensed under a Creative Commons: Attribution-NonCommercial-NoDerivatives 4.0 International (CC BY-NC-ND 4.0) License. 
Originally published at:

Schneider, Jörg; Eisenegger, Mark (2016). Wie Mediennutzer in die Welt schauen: Die Newsrepertoires der Schweizerinnen und Schweizer und ihre Themenagenden. Basel: Schwabe. 


\section{Qualität der Medien}

Wie Mediennutzer in die Welt schauen: Die Newsrepertoires der Schweizerinnen und Schweizer und ihre Themenagenden

Schweiz Suisse Svizzera 
Diese Studie erscheint als ePublikation im Zusammenhang mit dem Jahrbuch Qualität der Medien 2016, herausgegeben vom fög - Forschungsinstitut Öffentlichkeit und Gesellschaft / Universität Zürich. Studie und Jahrbuch sind zu beziehen unter www.schwabeverlag.ch.

Die ePublikation verfügt u.a. über folgende Funktionen: Volltextsuche und Verlinkungen zu Internetseiten.

Copyright @ 2016 Schwabe AG, Verlag, Basel, Schweiz, und fög - Forschungsinstitut Öffentlichkeit und Gesellschaft / Universität Zürich

Dieses Werk ist urheberrechtlich geschützt. Das Werk einschliesslich seiner Teile darf ohne schriftliche Genehmigung des Verlages und des fög - Forschungsinstitut Öffentlichkeit und Gesellschaft / Universität Zürich in keiner Form reproduziert oder elektronisch verarbeitet, vervielfältigt, zugänglich gemacht oder verbreitet werden.

Gestaltung: Thomas Lutz, Schwabe

Gesamtherstellung: Schwabe AG, Druckerei, Muttenz/Basel, Schweiz

ISBN 978-3-7965-3653-3

ISSN 2296-5114

rights@schwabe.ch

www.foeg.uzh.ch

www.schwabeverlag.ch 
Studien

2/2016

\section{Qualität der Medien}

Schweiz - Suisse - Svizzera

Wie Mediennutzer in die Welt schauen:

Die Newsrepertoires der Schweizerinnen und Schweizer und ihre Themenagenden

Jörg Schneider, Mark Eisenegger

Herausgegeben vom

fög - Forschungsinstitut Öffentlichkeit und Gesellschaft / Universität Zürich im Auftrag der Kurt Imhof Stiftung für Medienqualität

Schwabe Verlag Basel 


\section{Zusammenfassung}

Die Frage, wie Mediennutzer in die Welt schauen bzw. welches Bild sie von der Welt haben, untersuchen wir anhand einer Langzeitstudie, die seit 2009 einmal jährlich die Mediennutzung der Bevölkerung in der Schweiz untersucht und die Mediennutzung mit der Agenda der intensiv verfolgten Themen verknüpft. Aus den Daten zur Mediennutzung von inzwischen über 27000 Befragten werden sogenannte Newsrepertoires gebildet. Newsrepertoires erfassen die Kombination und die Nutzungsintensität der verschiedenen Medienangebote, die von einer einzelnen Person zu Informationszwecken konsumiert werden. Aus den individuellen Newsrepertoires lassen sich sechs Repertoiretypen aggregieren und soziodemografisch verorten. Stark in der traditionellen Medienwelt verhaftet sind die Repertoiretypen «Homeland Oriented», «Old World Boulevard» und «Old World \& Online», wobei der letztgenannte Typ die grösste Öffnung hin zur neuen, digitalisierten Medienwelt aufweist. In beiden Welten ist der Repertoiretyp der «Intensivnutzer» verankert, der sowohl traditionelle als auch online vermittelte Newsangebote ausgiebig in Anspruch nimmt. Am stärksten in der neuen Medienwelt beheimatet sind die Repertoiretypen der «Global Surfer» und «News-Deprivierten». Es zeigt sich, dass diese beiden Typen in den letzten Jahren einen massiven Zuwachs verzeichnen und inzwischen die Mehrheit der Bevölkerung repräsentieren. Das ist deshalb bedenklich, weil in diesen beiden Newsrepertoires die konsumierte Medienqualität abfällt, d.h. eine unterdurchschnittliche Qualitätsbilanz zu konstatieren ist. Die «News-Deprivierten» sind heute sogar die grösste Nutzungsgruppe überhaupt. Es handelt sich dabei um Nutzerinnen und Nutzer, die professionelle Newsangebote weit unterdurchschnittlich oft nutzen, und wenn, dann vor allem solche von minderer Qualität. Vor allem junge Frauen unter 30 Jahren sind in diesem Newsrepertoire überdurchschnittlich oft vertreten. Die Effekte der unterschiedlichen Newsrepertoires und der konsumierten Newsqualität zeigen sich in den Themenagenden, die die Repertoiretypen stark beachten. Die «Global Surfer» bevorzugen eine international ausgerichtete Themenagenda, aus der schweizerischen Medienarena ziehen sie sich zurück. Die «News-Deprivierten» nehmen vor allem Einzelereignisse und Bedrohungsszenarien wahr. Den Gegenpol zu diesem Repertoiretyp bilden die «Intensivnutzer», die aufgrund ihrer breiten Nutzung von qualitativ hochwertigen Informationsmedien eine vielfältige Themenagenda aufweisen und insbesondere auch Themenzusammenhänge wahrnehmen.

\section{Newsrepertoires als Instrumente der Medien- wirkungsforschung}

Im Zuge der Digitalisierung wächst die Anzahl der Medienangebote durch die Vervielfachung der Inhalte und die Ausdifferenzierung der Formate, in denen diese Inhalte verbreitet werden. Die Angebote konkurrieren somit unter den Bedingungen einer verschärften Aufmerksamkeitsökonomie um die Nutzer. Die Nutzer sehen sich ihrerseits aufgefordert, Strategien und Muster der Medienauswahl auszuprägen, um angesichts der vielfältigen Optionen und der eigenen beschränkten Zeitbudgets ihre Mediennutzung zu organisieren (Frank 2007, Webster 2014). Als Resultat dieser Prozesse differenziert sich die Mediennutzung entlang der individuellen Bedürfnisse und Präferenzen immer weiter aus, indem Medienangebote unterschiedlich stark genutzt und individuell miteinander kombiniert werden. Das Publikum fragmentiert sich, getrieben von selbstgewählten Mediennutzungsmustern, und wird zum multiplen Publikum (Hasebrink 2008). Doch die freie Wahl des Angebots hat ihre Grenzen: Nicht alle Angebote sind gratis verfügbar. Gerade Qualität hat ihren Preis. Und die Kompetenz zur Nutzung der neuen Onlineangebote ist vor allem über die Alterskohorten unterschiedlich verteilt. Ebenso mündet die Fragmentierung nicht in einer totalen Vereinzelung der Nutzer: Inhaltliche und formale Präferenzen sind nach wie vor stark milieuspezifisch vermittelt. Ökonomische Ressourcen und kulturelles Kapital bleiben also weiterhin relevante Faktoren, die den Rahmen abstecken, in dem die individuellen Mediennutzungsmuster entwickelt werden (Weiß 2009a, Weiß 2009b).

Das Konzept der Medienrepertoires bietet ein leistungsstarkes Instrument zur Erfassung der unter- 
schiedlichen Mediennutzungsmuster und der Nutzungstypen, die sich anhand ähnlicher Muster voneinander abgrenzen lassen. Medienrepertoires gehen nicht von einzelnen Medien, sondern von einzelnen Mediennutzern aus. Durch diesen personenzentrierten Zugriff wird das gesamte Spektrum der individuell genutzten Medien in den Blick genommen. Die Mediennutzung lässt sich so ausserdem in die Lebenswelt der Nutzerinnen und Nutzer einbetten. Motive und Bedürfnisse, Auswahlentscheidungen und ritualisierte Praktiken der Mediennutzung werden ebenso transparent wie soziodemografische und milieuspezifische Einflüsse (Hasebrink/Popp 2006, Weiß 2010).

Medienrepertoires sollen in der vorliegenden Studie aber nicht nur als beschreibendes Instrument dienen, um Nutzungsmuster zu erfassen und lebensweltlich zu verorten. Medienrepertoires sollen darüber hinaus als analytisches Instrument der Medienwirkungsforschung eingesetzt werden, um allfällige Effekte unterschiedlicher Mediennutzungen aufzudecken und zu erklären. In der vorliegenden Studie interessiert vor allem, inwieweit Medienrepertoires einen Beitrag leisten können, um die Themenagenden der Bevölkerung zu erklären. Als Themenagenda bezeichnen wir das Set von Themen, über das in der Öffentlichkeit innerhalb eines bestimmten Zeitraums verhandelt wird. Dieses öffentlich debattierte Themenset spiegelt sich auf der Nutzerseite in der individuellen Agenda derjenigen Themen wider, die von einer Person beachtet werden (Rössler 1997).

Information, Unterhaltung und Socializing sind als mögliche Funktionen der Mediennutzung zunehmend miteinander verschränkt. Gleichwohl scheint es geboten, die analytische Trennung beizubehalten, um die für die Fragestellung relevante Mediennutzung definieren zu können. Im Hinblick auf unsere Frage nach den Effekten auf die individuellen Themenagenden der Mediennutzer konkretisieren wir das Konzept der Medienrepertoires deshalb, indem wir uns auf die Informationsfunktion der Mediennutzung fokussieren. Genauer gesagt beschränken wir uns in der Repertoirebildung explizit auf Informationsmedien bzw. die Nutzung von News. In diesem Sinne sprechen wir nicht von Medienrepertoires, sondern konkreter von Newsrepertoires. Aus den Newsrepertoires, die wir zur Erfassung der individuellen Newsnutzungsmuster bilden, aggregieren wir sogenannte Repertoiretypen, die unser methodischer Zugriff auf die Nutzungstypen sind.

Hinsichtlich der Newsnutzung werden in der Forschung vor allem zwei gegensätzliche Typen beschrieben: Auf der einen Seite «News Seekers», die als Vielnutzer die ganze Palette der traditionellen und neuen Newsangebote konsumieren, und auf der anderen Seite «News Avoiders», die Informationen gezielt ausweichen und stattdessen ihre Aufmerksamkeit auf reine Unterhaltungsangebote lenken (Ksiazek/Malthouse/ Webster 2010, Strömbäck/Shehata 2010). Bei beiden Typen sind signifikante Zuwachsraten festzustellen. Daraus wird abgeleitet, dass sich bei der Verteilung von politischer Information eine Wissenskluft auftut. Diese Kluft setzt sich wiederum in unterschiedlichen Graden der politischen Partizipation fort (Bonfadelli 2002, Stroud 2011). Mit den Newsrepertoires möchten wir die Segmentierung der Newsnutzer in der vorliegenden Studie differenzierter erfassen. Es sollen nicht nur gegensätzliche Nutzungstypen betrachtet werden, die entlang einer Dimension, der Nutzungsintensität, definiert sind. Vielmehr soll empirisch explorativ die Vielfalt der Nutzungsmuster zwischen diesen Polen aufgedeckt werden, die sich durch die vielfältigen Kombinationsmöglichkeiten der verschiedenen traditionellen Medien und neuen Onlineangebote ergeben. Damit schaffen wir die Möglichkeit, die Mediennutzungstypen nicht nur nach der Intensität ihrer Mediennutzung zu beurteilen. Zusätzlich können dann andere Dimensionen der Newsnutzung berücksichtigt werden, beispielsweise die Qualität der Newsnutzung, insofern die Nutzer mit verschiedenen Newsangeboten tatsächlich auch unterschiedliche Newsqualitäten konsumieren.

Wir gehen davon aus, dass die Repertoiretypen Auswirkungen darauf haben, wie die Nutzerinnen und Nutzer die Gesellschaft wahrnehmen. Selbst wenn wir nicht wie Niklas Luhmann eine Exklusivität behaupten, gemäss der wir alles, was wir über die Gesellschaft, ja die Welt, in der wir leben, wissen, durch die Medien wissen (Luhmann 2009), so sehen wir doch insbesondere die Massenmedien als zentrale Instanz der Vermittlung von gesellschaftlichen Themen und Ereignissen. Wenn nun die Massenmedien an Definitionskraft einbüssen, weil immer mehr Medien um die Aufmerksamkeit der Nutzer konkurrieren, die ihrerseits unterschiedliche Nutzungsmuster ausprägen, zersplit- 
tert dann das Wissen über die Gesellschaft? Dies wäre einerseits der Fall, wenn die verschiedenen Medienangebote tatsächlich unterschiedliche Themensetzungen, Deutungsmuster und Newsqualitäten offerierten. Andererseits könnte auch die Medienangebotsauswahl der Nutzerinnen und Nutzer über die Wissensbestände entscheiden, indem die Nutzer gezielt bestimmte Themen und Deutungsmuster ansteuern und alternative Inhalte meiden. Dass solche Schliessungsprozesse durch die Selektionsalgorithmen der Suchmaschinen und Social-Media-Plattformen befördert werden, wird mit dem Begriff der «Echokammern» bzw. «selective exposure» (Stroud 2010, Pariser 2011, Levendusky 2013) beschrieben.

In der vorliegenden Studie werden wir zunächst die Datengrundlage und das methodische Vorgehen erläutern (Kapitel 2). Danach stellen wir die sechs Repertoiretypen vor, die ähnliche Newsrepertoires zusammenfassen und anhand ihrer Mediennutzungsprofile beschrieben werden (Kapitel 3). Für die Newsrepertoires werden Qualitätsbilanzen berechnet, um die Newsqualität zu bestimmen, die die Repertoiretypen nutzen (Kapitel 4). Anhand von soziodemografischen Variablen lassen sich die Repertoiretypen in den Lebenswelten verankern (Kapitel 5), um schliesslich ihre Anteilsentwicklung in den vergangenen acht Jahren nachzuzeichnen (Kapitel 6). Abschliessend geht es um die Frage, wie die Mediennutzer als Folge ihres Medienkonsums in die Welt schauen. Dazu werden die Unterschiede in den Themenagenden analysiert, um aufzuzeigen, wie die Newsrepertoires die Beachtung von Themen und Ereignissen prägen und somit die Welt- und Gesellschaftswahrnehmung der Nutzerinnen und Nutzer auf spezifische Weise einfärben (Kapitel 7).

\section{Datengrundlage und methodisches Vorgehen}

In einer alljährlich durchgeführten Mediennutzungsstudie des fög in Zusammenarbeit mit GfK Switzerland werden seit 2009 rund 3400 Onlineinterviews durchgeführt. Die Grundgesamtheit, die durch die Befragung erreicht werden soll, ist die Wohnbevölkerung in der Deutschschweiz und der Suisse romande im Alter zwischen 16 und 69 Jahren. Innerhalb dieser Grundgesamtheit wird die Bevölkerung fokussiert, die $\mathrm{Zu}-$ gang zum Internet hat und im Sinne des weitesten
Nutzerkreises regelmässig im Internet surft. Damit sind rund 90\% der Schweizer Wohnbevölkerung abgedeckt. Die Stichprobe wird anhand der Strukturdaten des BfS zur Internetnutzung nach Geschlecht und Alter gewichtet. Sie ist somit repräsentativ für die grosse Mehrheit der Schweizer Bevölkerung, die das Internet nutzt. Bei einem Vertrauensniveau von 95\% beträgt die Fehlertoleranz der Stichprobe 1,7\%. Der Kern der Befragung ist seit 2009 unverändert, sodass die Datenreihe inzwischen über acht Jahre reicht und insgesamt über 27000 Interviews umfasst.

Diese Befragung mit ihrer jährlich wiederholten Erhebung bietet für die vorliegende Studie eine ausgezeichnete Datengrundlage, um die Mediennutzung der Schweizer Bevölkerung zu erfassen und die Effekte unterschiedlicher Nutzungsmuster über lange Zeiträume zu analysieren. Es lässt sich nachzeichnen, wie sich die Mediennutzung im Zuge der Digitalisierung in den letzten Jahren verändert hat. Dabei zeigt sich, dass die News, die aufgrund der genutzten Informationsmedien rezipiert werden, massive Qualitätsunterschiede aufweisen. Dies hat belegbare Effekte auf die Wahrnehmung von Gesellschaft in Form der beachteten Themen und Ereignisse.

Im Zentrum der Befragung steht die Mediennutzung zu Informationszwecken: Welche Medientypen und welche Medientitel nutzen die Befragten, um sich über Themen und Ereignisse zu informieren? Welche Medien sind ihre Hauptquellen für News? Dabei wird das gesamte Spektrum der «alten» und «neuen» Medienwelt abgefragt, von den traditionellen Mediengattungen Radio, Fernsehen und Presse, wobei zwischen unterschiedlichen Typen wie Abonnements-, Boulevard- und Pendlerpresse unterschieden wird, bis hin zu den Online-Newsangeboten der Medienhäuser und den via Social-Media-Plattformen konsumierten News.

Anhand dieser Mediennutzungsdaten lassen sich Newsrepertoires bilden. Für jeden Befragten wird das individuelle Repertoire bestimmt, das er sich aus dem Angebot unterschiedlicher Newsmedien zusammenstellt, um sich zu informieren. Befragte mit ähnlichen Newsrepertoires werden induktiv durch Clusteranalysen zu charakteristischen Repertoiretypen zusammengefasst. Dabei werden in einem iterativen Algorithmus die Nutzerinnen und Nutzer jeweils einem Cluster zugeordnet, wobei möglichst hohe Ähnlichkeit der Nutzer 
innerhalb des Clusters und möglichst grosse Unterschiedlichkeit zwischen den Clustern angestrebt werden. Über die acht Untersuchungsjahre von 2009 bis 2016 haben sich jeweils sechs stabile Repertoiretypen etabliert.

Für jedes individuelle Newsrepertoire und jeden aggregierten Repertoiretyp lässt sich eine Qualitätsbilanz errechnen. Diese zeigt, wie intensiv und vielfältig die Nutzer Informationsmedien konsumieren und mit welcher Qualität sie dabei versorgt werden. Die Qualitätsbilanz ist messtechnisch das Produkt der Intensität der Newsmediennutzung einerseits sowie der Medienqualität des jeweils genutzten Repertoires andererseits. Je mehr und je intensiver Medien von hoher Qualität genutzt werden, desto höher ist die Qualitätsbilanz. Zur Quantifizierung der Medienqualität wird die Berichterstattung der relevanten schweizerischen Medientitel mittels Inhaltsanalysen erfasst. Aus einem normativen, demokratietheoretischen Qualitätsverständnis werden vier Qualitätsdimensionen abgeleitet: Relevanz, Vielfalt, Einordnungsleistung und Professionalität sowie anhand von mehreren Indikatoren inhaltsanalytisch erhoben und gescort (fög 2016). Die Qualitätsscores der einzelnen Medientitel und der zusammengefassten Medientypen können herangezogen werden, um die Newsrepertoires im Hinblick auf ihre journalistische Qualität zu beurteilen. So lässt sich bestimmen, welche Newsqualität die Repertoiretypen aufweisen.

Die Bildung der Repertoiretypen erfolgt ausschliesslich aufgrund der Mediennutzung $\mathrm{zu}$ Informationszwecken. Anschliessend werden die Repertoiretypen soziodemografisch verortet, um zu verdeutlichen, in welche sozialen Kontexte die Newsrepertoires typischerweise eingelagert sind. In Bezug auf die insgesamt sechs gebildeten Repertoiretypen zeigen sich klare Unterschiede im Hinblick auf Alter, Geschlecht, Bildung und Erwerbstätigkeit, aber auch zwischen Stadt- und Landbevölkerung, zwischen den Sprachregionen sowie zwischen Schweizern und Ausländern. Diese Unterschiede helfen dabei, die Repertoires deskriptiv zu verorten und entlang dieser Merkmale zu typisieren.

Die Befragungen finden jeweils zu Jahresbeginn statt. Gefragt wird nicht nur nach der Mediennutzung, sondern auch nach Themen und Ereignissen, die die Befragten im vorangegangenen Jahr besonders intensiv verfolgt haben. So lässt sich die persönliche Themen- agenda (in Bezug auf die Themen, die in der medial vermittelten Öffentlichkeit verhandelt wurden) ermitteln, die im Untersuchungsjahr bestimmend war. $\mathrm{Zu}$ diesem Zweck wird den Befragten eine Liste mit 20 Themen und Ereignissen vorgelegt, die in der Schweizer Medienarena besonders viel Resonanz erzielten. Dabei wird auf die kontinuierlich stattfindende Erhebung der Berichterstattung in den Schweizer Leitmedien durch das fög zurückgegriffen, die systematisch die Kommunikationsereignisse in der schweizerischen Medienöffentlichkeit erfasst (www.foeg.uzh.ch/ de/analyse/medienagenda.html). Aus diesen 20 TopKommunikationsereignissen mit besonders grossem Berichterstattungsvolumen im Untersuchungsjahr wählen die Befragten diejenigen fünf Ereignisse aus, die sie selbst am intensivsten verfolgt haben. Anhand dieser Auswahl können die Themenagenden der Repertoiretypen mittels Kreuztabellierungen und standardisierter Residuen auf über- bzw. unterdurchschnittliche Aufmerksamkeit für einzelne Kommunikationsereignisse hin analysiert werden.

\section{Die Newsrepertoires der Newsnutzer in der Schweiz}

Die Schweizer Bevölkerung lässt sich anhand ihrer individuellen Newsrepertoires in sechs Repertoiretypen unterteilen. Hinter diesen Typen stehen viele unterschiedliche Nutzerinnen und Nutzer, die allein aufgrund der Tatsache, dass sie die gleichen Medien in ähnlicher Intensität nutzen, um sich über News zu informieren, in einem Cluster zusammengefasst wurden. Gleichwohl erscheint es sinnvoll, ein Bild zu entwerfen, das die Repertoiretypen in der Lebenswelt verankert, indem typische Merkmale hervorgehoben werden, beispielsweise im Hinblick auf das Milieu, die Berufstätigkeit, das Alter oder das Geschlecht (vgl. Darstellung 1). Damit ist nicht gesagt, dass die Nutzerinnen und Nutzer eines Repertoiretyps allesamt zwangsläufig diesem Milieu, diesem Tätigkeitsfeld, dieser Altersgruppe und diesem Geschlecht angehören. Es sind vielmehr statistisch gesprochen überzufällige Häufungen der entsprechenden persönlichen Merkmale, die sich in den Typen finden. Auf der Basis dieser Informationen illustrieren wir die Repertoiretypen anhand von Bildern und zeichnen einen Steckbrief, der die Nutzungsmotive und Verhaltensweisen der Typen veranschaulicht. 


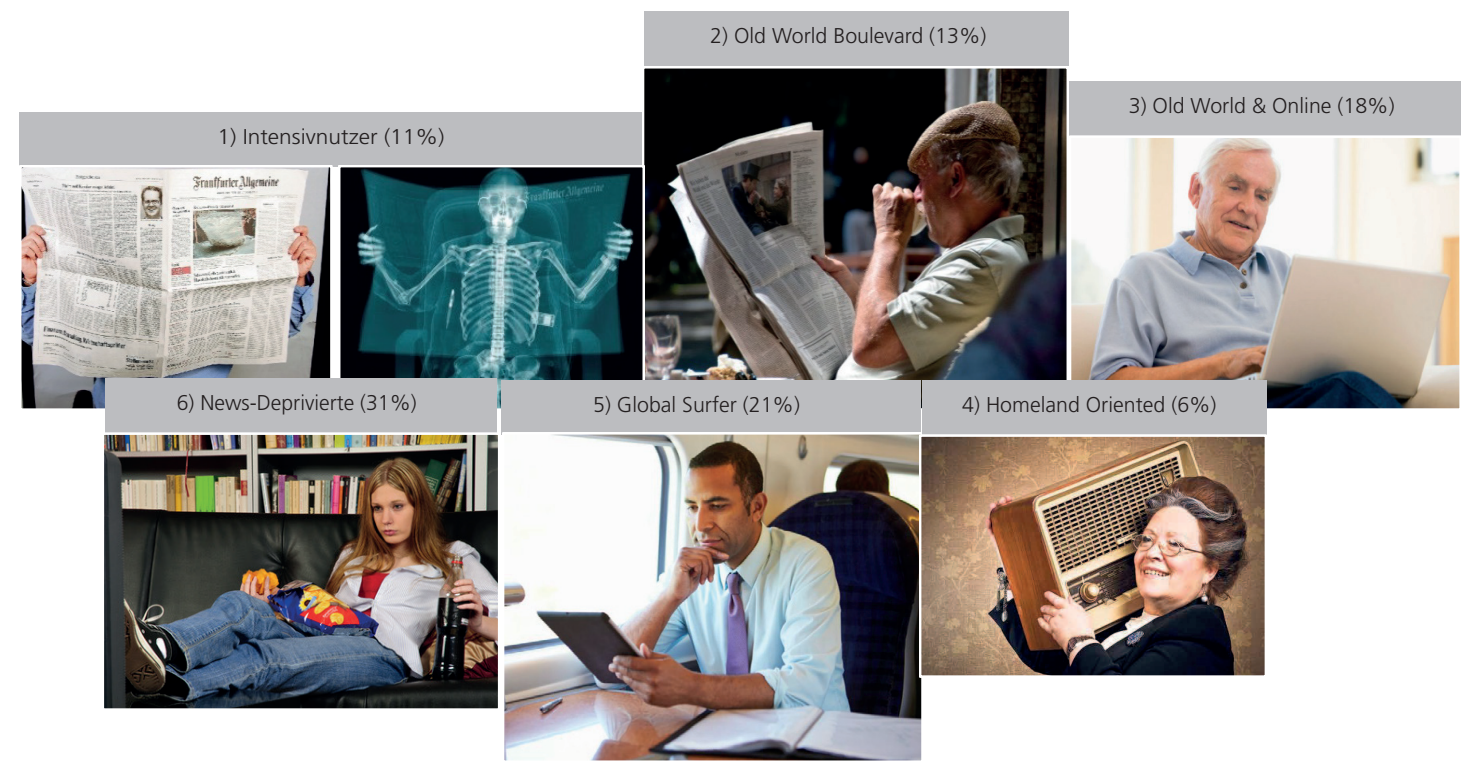

Darstellung 1: Repertoiretypen - Typenbilder

\section{Sechs Repertoiretypen - ein Überblick anhand von Typenbildern}

(1) Der Typ «Intensivnutzer» nutzt ein sehr breites Spektrum von traditionellen und neuen Medien, um sich über Nachrichten zu informieren. Der Blick in die Qualitätszeitung scheint nicht zuletzt ein Statussymbol zu sein, um seine gehobenen Ansprüche zur Schau zu stellen. Für jede Medienprofessorin ist er das Idealbild des kompetenten Newsrezipienten mit hohem Qualitätsanspruch. Und für jeden Medienmacher verkörpert er die Wunschvorstellung des zahlungsbereiten Newskonsumenten. Allerdings bekommt er zunehmend geisterhafte Züge: Alle suchen ihn, aber nur wenige können ihn an ihr Angebot binden.

(2) Der Typ «Old World Boulevard» nutzt vor allem traditionelle Medien, und hier insbesondere die Boulevardzeitung, um sich zu informieren. Wenn im Café die Boulevardzeitungen vergeben sind, dann nimmt er auch einmal die Qualitätszeitung in die Hand. Die blättert er aber rasch durch, um schliesslich bei den vermischten Nachrichten und den Sportergebnissen zu verweilen. Diesen Typ finden wir eher am Lac Léman und weniger am Zürichsee.
(3) Die Newsrepertoires der «Old World \& Online» ergänzen das traditionelle Set von Newsmedien mit ausgewählten Angeboten aus dem Internet. Diese Mediennutzer sind keine Digital Natives, sondern haben sich von ihren Kindern erklären lassen, wie die Nutzung von Onlinenews funktioniert. Inzwischen nutzen sie die Möglichkeiten der neuen digitalen Welt mit grosser Freude. Das Abonnement der regionalen Tageszeitung haben sie aber trotzdem nicht abbestellt.

(4) Die Nutzerinnen und Nutzer, die dem Typ «Homeland Oriented» angehören, nutzen das Internet eher, um Kochrezepte zu suchen. Ansonsten verlassen sie sich auf die bewährten Medien, um sich zu informieren: morgens ein kurzer Blick in die Tageszeitung, tagsüber im Hintergrund die Radionachrichten, abends ritualisiert die Regionalnachrichten im Privatfernsehen oder die Tagesschau im Schweizer Fernsehen.

(5) Der Typ «Global Surfer» steht im Berufsleben und hat viel zu tun. Über PC, Laptop und Mobile ist er im Grunde rund um die Uhr an den Nachrichtenfluss angeschlossen. Das Internet mit den vor allem kostenfreien Newsangeboten ist für ihn die zentrale Informationsquelle. Internationale Medien gehö- 


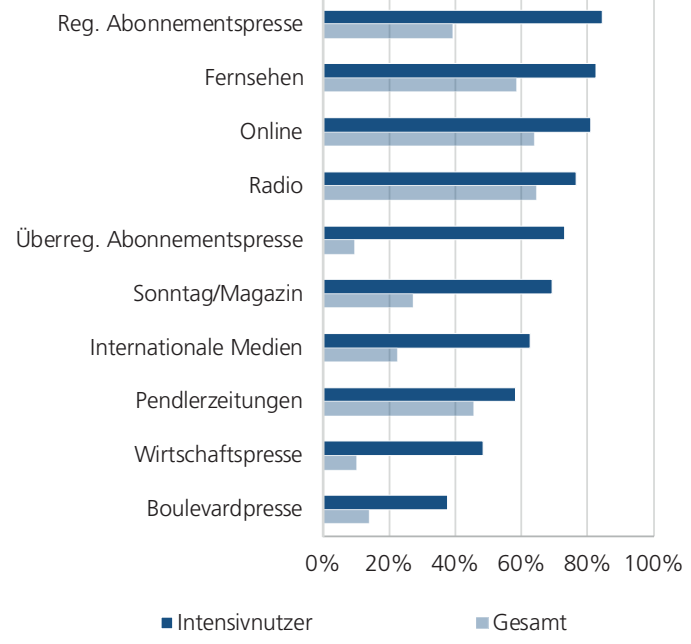

Darstellung 2: «Intensivnutzer» - für Informationszwecke genutzte Medientypen

Dargestellt ist der Anteil der regelmässigen Nutzer des jeweiligen Medientyps (Kategorien «oft» und "sehr oft» auf einer 5-stufigen Likert-Skala) im Vergleich zum Durchschnitt der Gesamtheit aller Befragten. Datengrundlage: Erhebung 2016 mit $\mathrm{n}=373$ Befragten des Repertoiretyps und $\mathrm{n}=$ 3477 Befragten insgesamt.

Lesebeispiel: 73\% der «Intensivnutzer» lesen die überregionale Abonnementspresse regelmässig, während dieser Medientyp im Schnitt von nur $10 \%$ der Befragten genutzt wird.

ren für ihn ebenfalls zum Repertoire. Die gedruckte Zeitung nimmt er nur dann in die Hand, wenn sie gratis in der S-Bahn oder im Tram bereitliegt.

(6) Mediennutzerinnen und -nutzer, die wir dem Typ «News-Deprivierte» zuschlagen, machen um News meist einen weiten Bogen. Ihr Themeninteresse ist punktuell und primär an Softnews orientiert, eine Zahlungsbereitschaft für News ist nicht vorhanden. Die «neue Medienwelt» ist ihr Ding, doch ihre Mediennutzung ist kaum ritualisiert. So schnell wie sie Angebote entdecken und exzessiv nutzen, so schnell sind sie gelangweilt und entschwinden in den Weiten des Internets. Die «News-Deprivierten» sind die grosse Herausforderung für die Medienmacher und die Mediennutzungsforscher.

\subsection{Profil des Repertoiretyps "Intensivnutzer»}

«Intensivnutzer» (vgl. Darstellungen 2 und 3) investieren von allen Repertoiretypen die meiste Zeit in den Konsum von News. Ihre Newsrepertoires decken ein

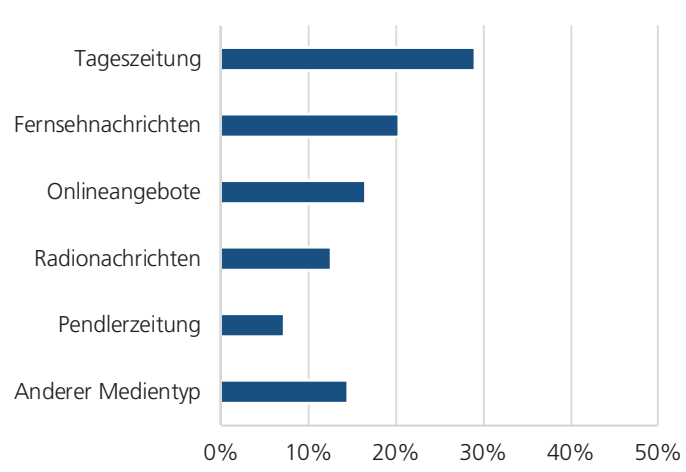

Darstellung 3: «Intensivnutzer» - Hauptquellen für News Dargestellt sind die fünf Hauptquellen für News in absteigender Relevanz (zusammen mit der Restkategorie «anderer Medientyp»: 100\%). Datengrundlage: Erhebung 2016 mit $n=373$ Befragten des Repertoiretyps. Lesebeispiel: Für 29\% der «Intensivnutzer» ist die Tageszeitung die Hauptquelle für News.

breites Spektrum von Medientypen ab. Alle Medientypen werden von ihnen intensiver als vom Durchschnitt aller Befragten genutzt. Die Repertoires beinhalten oft mehrere regionale und überregionale Tages-, Wochen- und Sonntagszeitungen, auch Onlinemedien werden ausgiebig genutzt. Als Hauptinformationsquelle dienen jedoch vor allem traditionelle Medien: Tageszeitung, Fernseh- und Radionachrichten. Ist das Internet ihre Hauptquelle, so sind es die professionellen Online-Newssites der etablierten Medienanbieter.

News gehören zum Alltag der «Intensivnutzer». Und die Nutzung von vielfältigen und qualitativ hochwertigen Angeboten gehört zum Selbstverständnis dieses Typs, der sich wohl als aufgeklärter, gesellschaftlich interessierter Zeitgenosse beschreiben würde. In den individuellen Newsrepertoires dieses Typs finden sich besonders häufig Medientitel, die traditionell als bildungsbürgerliche Statussymbole gelten, wie die Qualitätstitel der überregionalen Abonnementspresse. Auch internationale Medien und Titel der Wirtschaftspresse werden von diesen Nutzern deutlich häufiger genutzt als von allen anderen Repertoiretypen. Einzig die Social-Media-Nutzung fällt vergleichsweise gering aus. Es ist zu vermuten, dass er diesen Zugangskanal zu Nachrichteninhalten weniger benötigt, da er bereits durch die originären Anbieter umfassend versorgt ist. Eventuell genügen die über Social-Media-Plattformen selektiv verbreiteten Inhalte auch nicht durchgehend 


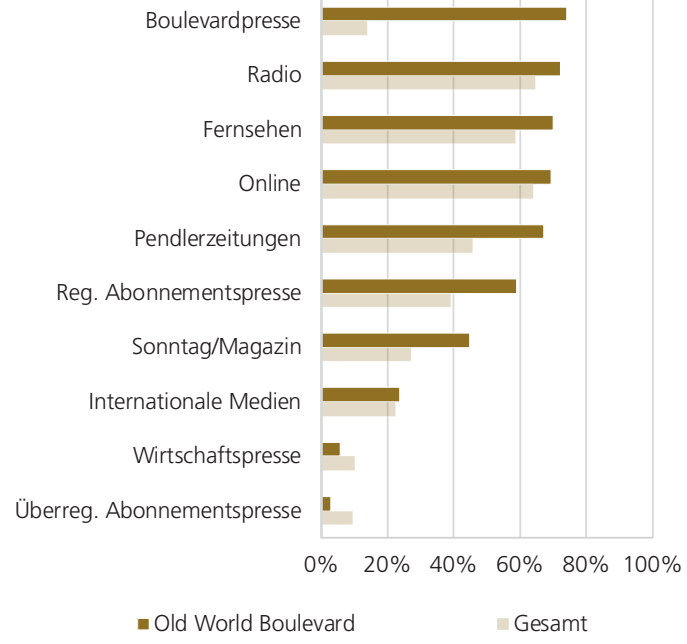

Darstellung 4: «Old World Boulevard» - für Informationszwecke genutzte Medientypen

Dargestellt ist der Anteil der regelmässigen Nutzer des jeweiligen Medientyps zu Informationszwecken (Kategorien "oft» und "sehr oft» auf einer 5-stufigen Likert-Skala) im Vergleich zum Durchschnitt der Gesamtheit aller Befragten. Datengrundlage: Erhebung 2016 mit $n=446$ Befragten des Repertoiretyps und $n=3477$ Befragten insgesamt. Lesebeispiel: 74\% der «Old World Boulevard» nutzen die Boulevardpresse regelmässig, während dieser Medientyp im Schnitt von nur 14\% der Befragten genutzt wird.

seinen hohen Qualitätsansprüchen. Gleichwohl ist er offen für neuartige Angebote, z.B. für unabhängige Newsblogs von hoher Qualität, die er häufiger als andere Nutzer in seinem Newsrepertoire hat.

\subsection{Profil des Repertoiretyps "Old World Boulevard"}

«Old World Boulevard» (vgl. Darstellungen 4 und 5) bezeichnet einen Repertoiretyp, der die traditionellen Medien Radio, Fernsehen und regionale Abonnementszeitung sowie Sonntagszeitungen und Wochenmagazine überdurchschnittlich intensiv nutzt. Daneben werden auch Onlineangebote und Pendlerzeitungen gerne ins Newsrepertoire aufgenommen. Das hervorstechende Kennzeichen dieses Repertoiretyps ist jedoch eindeutig die Boulevardzeitung. Deren Nutzung kommt in diesem Repertoire fünfmal häufiger als im Durchschnitt vor. Durch diesen Umstand hebt sich der Typ «Old World Boulevard» von den «Intensivnutzern» ab, deren Boulevardnutzung viel geringer ist. Anstelle der Boulevardzeitung haben Letztere

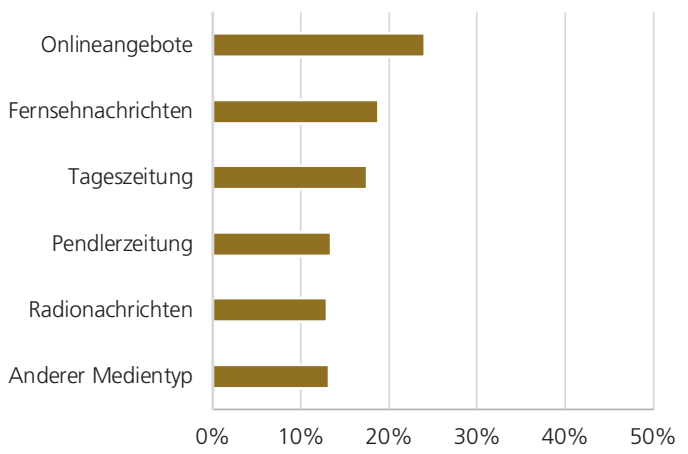

Darstellung 5: «Old World Boulevard» - Hauptquellen für News Dargestellt sind die fünf Hauptquellen für News in absteigender Relevanz (zusammen mit der Restkategorie «anderer Medientyp»: 100\%). Datengrundlage: Erhebung 2016 mit n = 446 Befragten des Repertoiretyps. Lesebeispiel: Für 19\% der «Old World Boulevard» sind Fernsehnachrichten die Hauptquelle für News.

eine überregionale Abonnementszeitung im Repertoire und ausserdem viel mehr internationale Medien und Wirtschaftstitel. Als Hauptquelle für News dienen dem stark in der alten Medienwelt verhafteten Typ «Old World Boulevard» neben den traditionellen Medien oft auch Onlineangebote. Auch hier sind es insbesondere die Newssites der traditionellen Boulevardzeitungen, die die Nutzerinnen und Nutzer dieses Typs frequentieren.

Das Lesen, Schauen und Hören von Nachrichten ist für die Vertreterinnen und Vertreter des Typs «Old World Boulevard» ein fester Bestandteil des Alltags. Medienkonsum scheint Ausdruck der Lebensqualität zu sein und dafür wird ein vergleichsweise hohes Zeitbudget investiert. Dieser Repertoiretyp nutzt ein vielfältiges Set von Medien, um sich über Nachrichten zu informieren. Er ist durchaus bereit, für Nachrichten zu bezahlen. Auch die hohe Intensität der Newsnutzung verweist auf ein hohes Newsinteresse, das sich allerdings vor allem auf Human Interest und Sport, d.h. auf Softnews, richtet. Die relativ ausgeglichene Nutzung der verschiedenen Medientypen und die annähernde Gleichverteilung bei den Hauptquellen deuten darauf hin, dass die Newsnutzung des Typs «Old World Boulevard» weniger an Formaten, sondern sehr stark an den «weichen» Themen orientiert ist. Als Maxime des Newskonsums würden diese Nutzerinnen und Nutzer wohl angeben, auf dem Laufenden bleiben zu wollen, um in ihrem Umfeld mitreden zu können. 


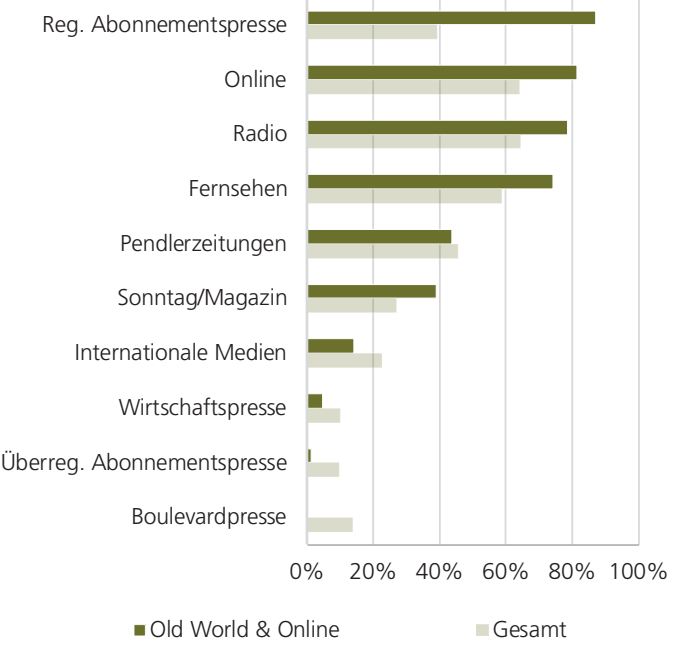

Darstellung 6: «Old World \& Online» - für Informationszwecke genutzte Medientypen

Dargestellt ist der Anteil der regelmässigen Nutzer des jeweiligen Medientyps zu Informationszwecken (Kategorien «oft» und «sehr oft» auf einer 5-stufigen Likert-Skala) im Vergleich zum Durchschnitt der Gesamtheit aller Befragten. Datengrundlage: Erhebung 2016 mit $n=643$ Befragten des Repertoiretyps und $n=3477$ Befragten insgesamt. Lesebeispiel: $87 \%$ der «Old World \& Online» nutzen die regionale Abonnementspresse regelmässig, während dieser Medientyp im Schnitt von nur $39 \%$ der Befragten genutzt wird.

\subsection{Profil des Repertoiretyps "Old World \& Online»}

Der Repertoiretyp «Old World \& Online» (vgl. Darstellungen 6 und 7) ist durch die überdurchschnittlich häufige Nutzung der regionalen Tageszeitung, aber auch durch die Nutzung von Onlinemedien charakterisiert. Insbesondere die Onlineseiten der klassischen Nachrichtenmedien werden neben den traditionellen Medien genutzt, wobei letztere nach wie vor die relevanten Newsquellen sind. Die Hauptnachrichtenquelle bleibt die regionale Abonnementszeitung. Überregionale Abonnements- und Boulevardzeitungen - die charakteristischen Merkmale der Typen «Intensivnutzer» und «Old World Boulevard» - spielen gar keine Rolle.

Die Newsnutzung des Typs «Old World \& Online» ist klar in den Tagesablauf eingebettet. Wichtige Nachrichtenquellen sind die regionale Tageszeitung, die seit vielen Jahren auf den Frühstückstisch und damit zum Newsrepertoire gehört, und die Onlineangebote von Zeitungen und Rundfunkanbietern im Internet, die

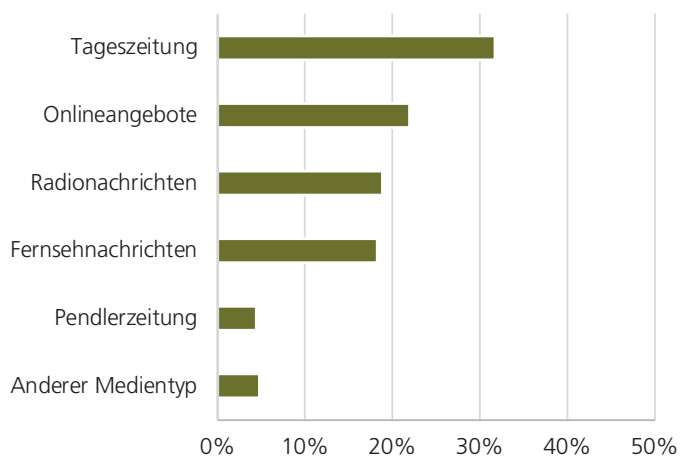

Darstellung 7: «Old World \& Online» - Hauptquellen für News Dargestellt sind die fünf Hauptquellen für News in absteigender Relevanz (zusammen mit der Restkategorie «anderer Medientyp»: 100\%). Datengrundlage: Erhebung 2016 mit $n=643$ Befragten des Repertoiretyps. Lesebeispiel: Für 32\% der «Old World \& Online» ist die Tageszeitung die Hauptquelle für News.

ebenfalls ins Repertoire integriert sind. Nicht nur für die traditionelle Zeitung wird gezahlt, auch für einzelne Onlineangebote ist man bereit, einen Betrag zu entrichten. Das Themenspektrum, das die Nutzerinnen und Nutzer dieses Repertoiretyps interessiert, ist wesentlich weiter gesteckt als beim Typ «Old World Boulevard». Es setzt einen Akzent auf Hardnews aus Politik, Wirtschaft und Kultur, ist aber auch offen für Softnews. Als Bezugsraum ist neben dem lokal-regionalen Geschehen vor allem die nationale Ebene von Interesse. Die ritualisierte Newsnutzung ist ausgeprägt. So hat die Tagesschau am Abend einen festen Platz im Nutzungsverhalten. Das Motto dieses Repertoiretyps könnte lauten: «Informiere Dich, damit Du über die relevanten Themen Bescheid weisst!»

\subsection{Profil des Repertoiretyps «Homeland Oriented»}

Der Typ «Homeland Oriented» (vgl. Darstellungen 8 und 9) zeichnet sich durch Newsrepertoires aus, die eine deutlich regionale Orientierung haben. Die wichtigsten Newsmedien sind die regionale Tageszeitung und die Regionalnachrichten in Radio und Fernsehen. Ausser diesen drei Medien sind praktisch keine anderen Newsangebote von Bedeutung. Das ist auch daran zu erkennen, dass sich bei den Hauptinformationsquellen hinter den drei Topplätzen, die von den genannten belegt werden, eine grosse Lücke auftut. Neuere Medientypen wie Onlineangebote von Zeitungen 


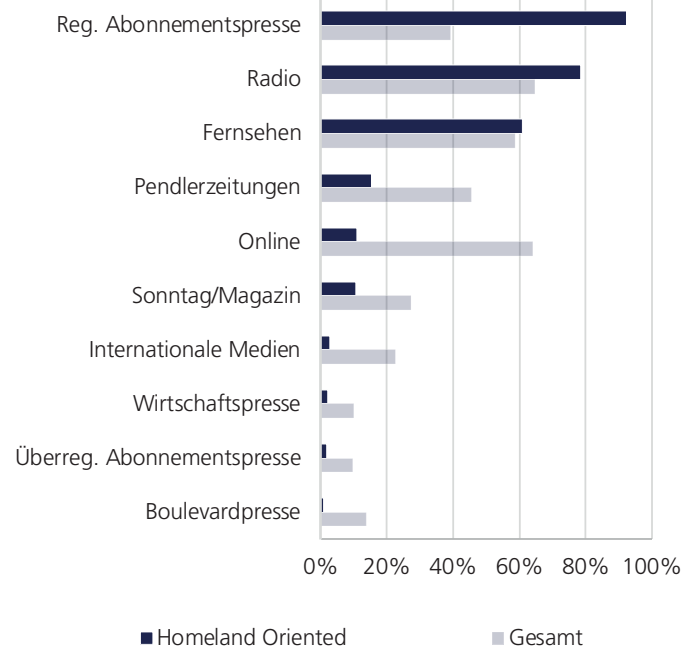

Darstellung 8: «Homeland Oriented» - für Informationszwecke genutzte Medientypen

Dargestellt ist der Anteil der regelmässigen Nutzer des jeweiligen Medientyps zu Informationszwecken (Kategorien «oft» und "sehr oft» auf einer 5-stufigen Likert-Skala) im Vergleich zum Durchschnitt der Gesamtheit aller Befragten. Datengrundlage: Erhebung 2016 mit $n=212$ Befragten des Repertoiretyps und $n=3477$ Befragten insgesamt. Lesebeispiel: 92\% der «Homeland Oriented» nutzen die regionale Abonnementspresse regelmässig, während dieser Medientyp im Schnitt von nur $39 \%$ der Befragten genutzt wird.

und Rundfunkanbietern, Pendlerzeitungen oder Social Media werden nur sehr selten genutzt.

Der Repertoiretyp der «Homeland Oriented» vereint Nutzerinnen und Nutzer, die sehr stark auf ein enges Set von traditionellen Newsmedien beschränkt sind. Diese ziehen sich gewissermassen in eine altbewährte Medienwelt zurück. Eine Öffnung hin zu neuen Medien oder Formaten findet praktisch gar nicht statt. Das Medienbudget enthält seit Jahren nur die Kosten für die Tageszeitung und die Billag. Es gibt keine klare thematische Interessenstruktur. Charakteristisch ist lediglich die Fokussierung des lokal-regionalen Nahbereichs und der Schweiz. Im Grunde zeigt dieser Repertoiretyp kein aktives Suchverhalten nach Nachrichten. Der Nachrichtenkonsum erfolgt wohl eher begleitend, eingebettet in den üblichen Tagesablauf. Die Nutzerinnen und Nutzer bekommen so nebenbei mit, was in der Schweiz und in ihrer Region geschieht.

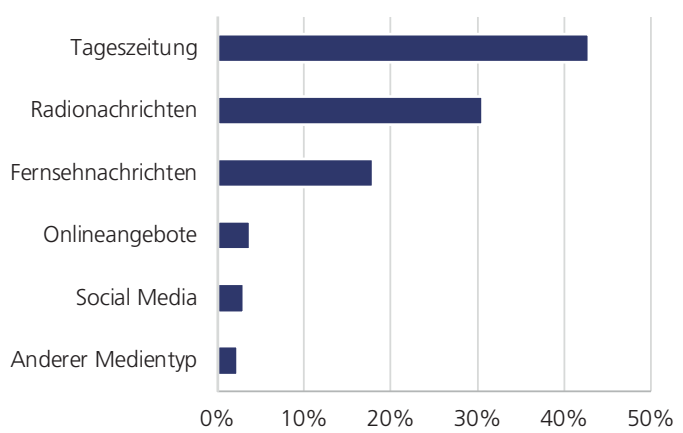

Darstellung 9: «Homeland Oriented» für News - Hauptquellen für News

Dargestellt sind die fünf Hauptquellen für News in absteigender Relevanz (zusammen mit der Restkategorie «anderer Medientyp»: 100\%). Datengrundlage: Erhebung 2016 mit $n=212$ Befragten des Repertoiretyps. Lesebeispiel: Für 43\% der «Homeland Oriented» ist die Tageszeitung die Hauptquelle für News.

\subsection{Profil des Repertoiretyps "Global Surfer"}

Der Repertoiretyp der «Global Surfer» (vgl. Darstellungen 10 und 11) informiert sich vor allem online und stärker als andere Nutzer über internationale Quellen. Die regelmässige Nutzung von Newssites im Internet ist überdurchschnittlich, ebenso sind die Onlineangebote von Zeitungen und Rundfunkanbietern mit Abstand die wichtigste Informationsquelle. Fernsehen und Radio ergänzen das Newsrepertoire. Stärker als bei anderen Typen werden internationale Medien als Newsquelle genutzt. Im Gegensatz dazu werden regionale und nationale Printmedien so gut wie überhaupt nicht konsumiert, weder Boulevard- noch regionale oder überregionale Abonnementszeitungen. Print wird praktisch nur in Form der kostenlosen Pendlerpresse in Anspruch genommen.

Der «Global Surfer» hat sich aus der alten Medienwelt der gedruckten Nachrichten weitgehend verabschiedet. Nachrichten im Radio oder Fernsehen nutzt er gerade noch durchschnittlich oft. Feste Strukturen der Newsnutzung im Alltag sind eher selten. Er konsumiert Nachrichten in den Zeitfenstern, die sich im Tagesablauf ergeben. Dieser Repertoiretyp sucht und findet seine Informationen im Internet, wobei er vor allem die Newssites der etablierten Medienmarken ansteuert. Häufig sind das Angebote aus dem Ausland. Auch Social Media und unabhängige Newsblogs werden von ihm genutzt. Allerdings verwendet er weniger Zeit auf 


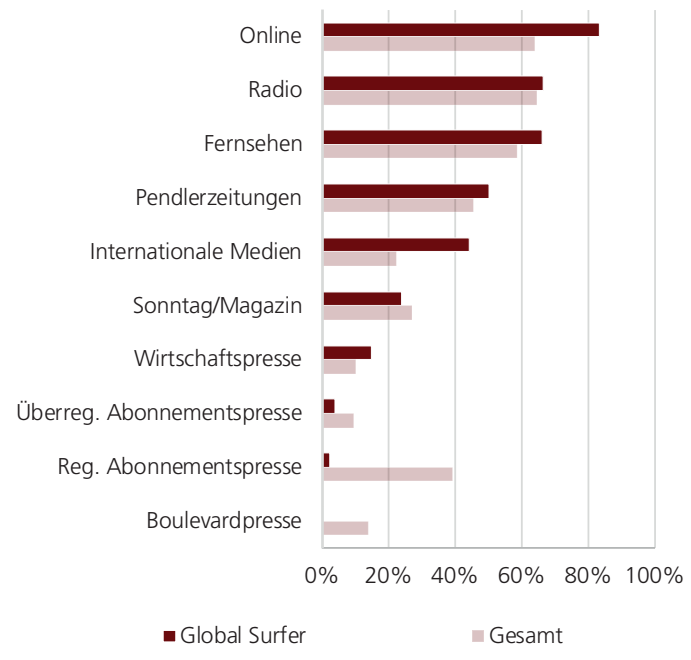

Darstellung 10: «Global Surfer» - für Informationszwecke genutzte Medientypen

Dargestellt ist der Anteil der regelmässigen Nutzer des jeweiligen Medientyps zu Informationszwecken (Kategorien «oft» und «sehr oft» auf einer 5-stufigen Likert-Skala) im Vergleich zum Durchschnitt der Gesamtheit aller Befragten. Datengrundlage: Erhebung 2016 mit $n=721$ Befragten des Repertoiretyps und $\mathrm{n}=3477$ Befragten insgesamt.

Lesebeispiel: $44 \%$ der "Global Surfer» nutzen internationale Medien regelmässig, während dieser Medientyp im Schnitt von nur 22\% der Befragten genutzt wird.

den Medien- und Newskonsum als andere Repertoiretypen. Für seichte Newskost nimmt er sich ebenso wenig Zeit wie für ausführliche Hardnews. Es fällt auf, dass der Typ «Global Surfer» Bezahlangebote äusserst selten nutzt. Wenn, dann bezahlt er allenfalls für News, die spezifische Themeninteressen bedienen, z.B. Wirtschaftszeitungen oder spezielle Wochenmagazine. Als Bezugsräume hat er weniger die Region oder die Schweiz im Blick, sondern er hat eine globale Perspektive. Seine Newsbedürfnisse würde er wohl so präzisieren: «Was? - Nur das, was mich interessiert. Wie? Schnell und auf den Punkt. Wann? - Zwischendurch, wenn ich Zeit dafür habe. Über welchen Kanal? - Statt analog lieber digital. Von wo? - Gerne aus dem Ausland.»

\subsection{Profil des Repertoiretyps "News-Deprivierte»}

«News-Deprivierte» (vgl. Darstellungen 12 und 13) investieren von allen Repertoires am wenigsten Zeit, um sich über Nachrichten zu informieren. Der Repertoire-

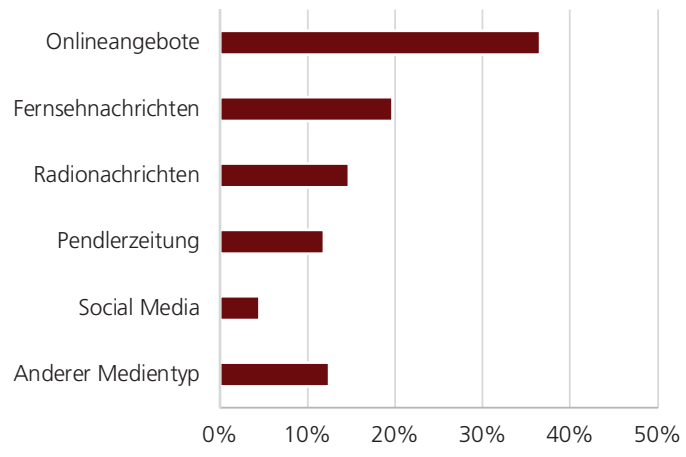

Darstellung 11: «Global Surfer» - Hauptquellen für News

Dargestellt sind die fünf Hauptquellen für News in absteigender Relevanz (zusammen mit der Restkategorie «anderer Medientyp»: 100\%). Datengrundlage: Erhebung 2016 mit $n=721$ Befragten des Repertoiretyps. Lesebeispiel: Für 37\% der «Global Surfer» sind die Onlineangebote von Zeitungen und Rundfunkanbietern die Hauptquelle für News.

typ der «News-Deprivierten» bündelt dementsprechend vor allem Personen, die sich durch eine unterdurchschnittliche Nutzung aller abgefragten Newsangebote auszeichnen und sich vor allem für Softnews interessieren. Insbesondere Printtitel, für die man bezahlen müsste, werden überhaupt nicht genutzt. Wenn die «News-Deprivierten» gedruckte Erzeugnisse mit Nachrichten in die Hand nehmen, dann nur in Form der Gratisangebote der Pendlerzeitungen. Auch die Onlineangebote von Zeitungen und Rundfunkanbietern werden von den «News-Deprivierten» seltener genutzt als von den anderen Repertoiretypen. Für $28 \%$ sind die Onlineangebote gleichwohl die Hauptquelle für News. Einzig der Anteil von Social Media als Hauptquelle ist bei ihnen mit 16\% grösser als bei allen anderen Repertoiretypen.

Der Repertoiretyp der «News-Deprivierten» versammelt Nutzerinnen und Nutzer, die sich wie selbstverständlich im Internet bewegen und die Angebote der «neuen Medienwelten» ausgiebig in Anspruch nehmen. Allerdings liegt ihr Fokus dabei eindeutig auf Unterhaltung und der Pflege von sozialen Kontakten. Nachrichten stehen nicht auf der Prioritätenliste. Die genutzten Angebote für News sind in der Regel kostenlos. Für News zu bezahlen, kommt diesen Nutzerinnen und Nutzern nicht in den Sinn. Ihre Selbstbeschreibung in Bezug auf Medien und Nachrichten könnte lauten: «Smartphone, Internet und Social Media sind mein Ding, News dagegen weniger.» 


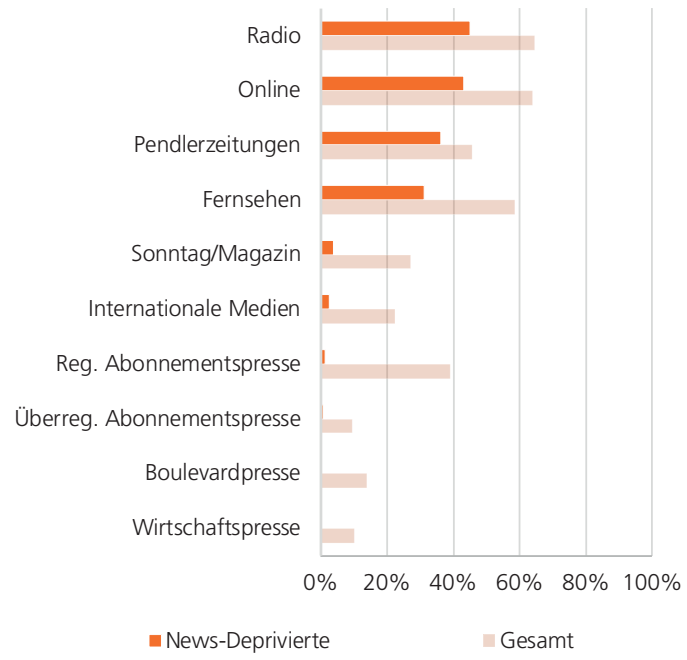

Darstellung 12: «News-Deprivierte» - für Informationszwecke genutzte Medientypen

Dargestellt ist der Anteil der regelmässigen Nutzer des jeweiligen Medientyps zu Informationszwecken (Kategorien «oft» und "sehr oft» auf einer 5-stufigen Likert-Skala) im Vergleich zum Durchschnitt der Gesamtheit aller Befragten. Datengrundlage: Erhebung 2016 mit n = 1083 Befragten des Repertoiretyps und $n=3477$ Befragten insgesamt.

Lesebeispiel: 36\% der «News-Deprivierten» nutzen Pendlerzeitungen regelmässig, während dieser Medientyp im Schnitt von 46\% der Befragten genutzt wird.

Dieser objektive Mangel an News wird durch den Begriff der «Deprivation» als sozial und gesellschaftspolitisch bedenklicher Zustand erfasst. Die charakteristische Unterversorgung mit News (geringe Ressourcen) geht wohl einher mit einer Verkümmerung der individuellen Fähigkeiten, News angemessen zu verarbeiten und in Abwägung unterschiedlicher Interpretationsangebote eine eigene Meinung auszubilden (geringe Kompetenzen). Es ist anzunehmen, dass sich dieser Repertoiretyp tendenziell aus der öffentlichen Sphäre zurückzieht (geringe gesellschaftliche und politische Partizipation) oder aber besonders anfällig für vereinfachende und irrationale Deutungsangebote ist.

\section{Qualitätsbilanzen - welche journalistische Qualität macht die Repertoiretypen aus?}

Um die Qualitätsbilanzen der Repertoiretypen anhand ihrer Newsrepertoires ermitteln zu können, müssen für jeden Befragten zwei Faktoren der Informations-

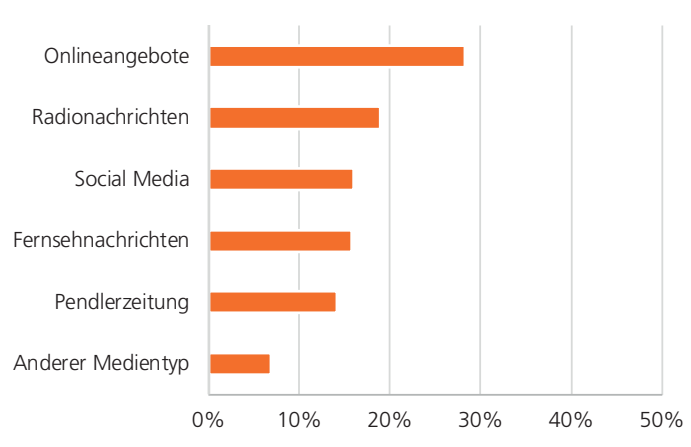

Darstellung 13: «News-Deprivierte» - Hauptquellen für News Dargestellt sind die fünf Hauptquellen für News in absteigender Relevanz (zusammen mit der Restkategorie «anderer Medientyp»: 100\%). Datengrundlage: Erhebung 2016 mit $n=1083$ Befragten des Repertoiretyps. Lesebeispiel: Für 28\% der «News-Deprivierten» sind die Onlineangebote von Zeitungen und Rundfunkanbietern die Hauptquelle für News.

mediennutzung betrachtet werden, die Güte sowie die Intensität der Newsnutzung (vgl. Darstellung 14).

Zum einen muss die genutzte Güte im jeweiligen Newsrepertoire bestimmt werden. Je grösser der Anteil der qualitativ guten Angebote, desto positiver fällt die Qualitätsbilanz auf der ersten Dimension aus. Deshalb wird für jeden Befragten die mittlere Qualität der genutzten Medienangebote, gewichtet mit deren Nutzungshäufigkeit, berechnet und auf eine Skala von 0 (ausschliesslich schlechte Qualität) bis 100 (ausschliesslich gute Qualität) transformiert. Die Qualität der individuellen Newsrepertoires wird anhand der inhaltsanalytischen Qualitätsscores für die genutzten Medientypen bestimmt.

Zum andern muss die Intensität der Newsnutzung in Anschlag gebracht werden. Denn eine intensive und breite Informationsnutzung erhöht die Wahrscheinlichkeit, auch mit qualitativ guten News in Berührung zu kommen. Wenn viele Informationsangebote genutzt werden, steigt die Chance, relevante, vielfältige, einordnende und professionelle News zu erhalten. Je intensiver die Newsnutzung (sowohl hinsichtlich der Nutzungshäufigkeit als auch der Breite des genutzten Angebots), desto stärker fliessen gute Angebote in die Qualitätsbilanz ein. Für jeden Befragten werden deshalb auf der zweiten Dimension die Häufigkeit und die Breite der genutzten Medienangebote quantifiziert und auf eine Skala von 0 (keine Newsnutzung) bis 100 (maximale Nutzung aller Medien) transformiert. 


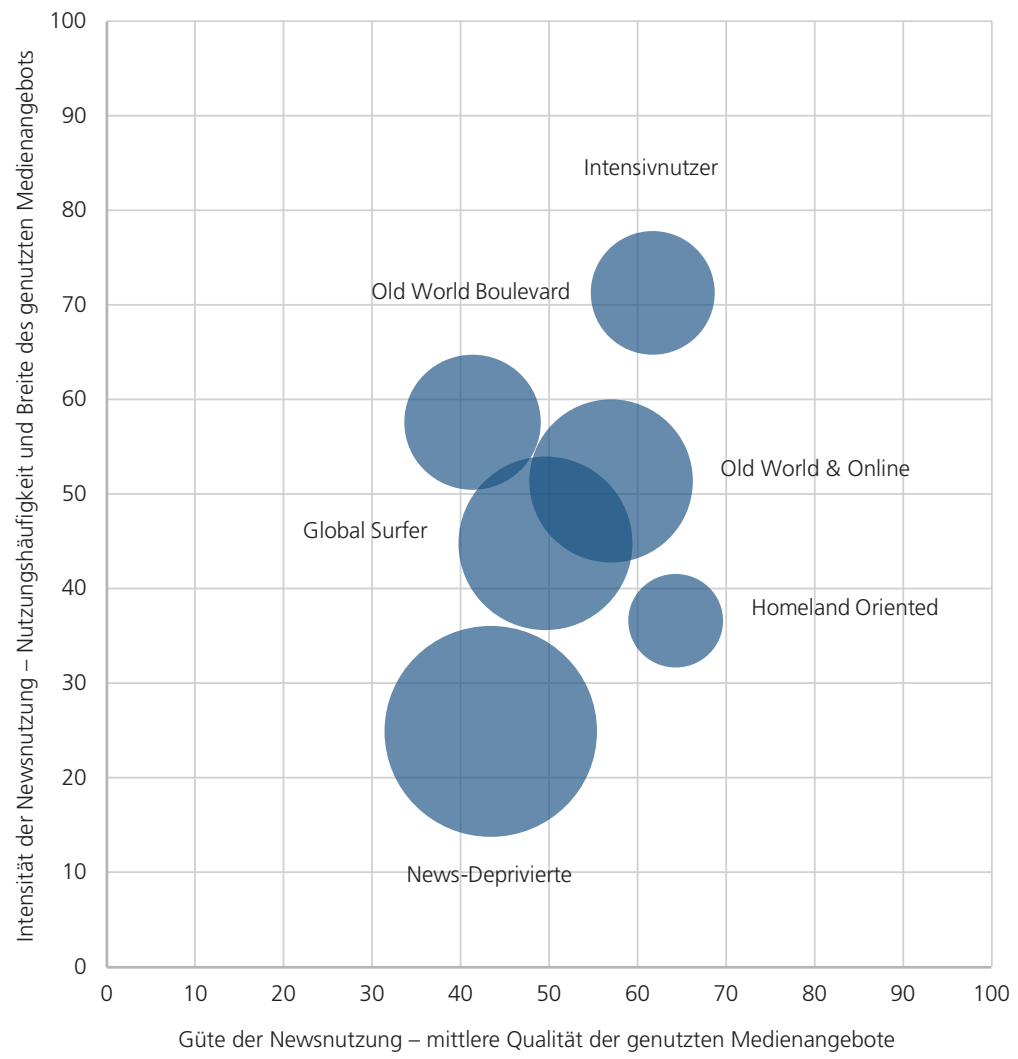

Darstellung 14: Repertoiretypen Güte der Newsnutzung und Intensität der Newsnutzung

Die Darstellung zeigt die mittlere Qualität der genutzten Medienangebote (horizontale Achse) sowie die Nutzungshäufigkeit und Breite des genutzten Medienangebots (vertikale Achse) für die sechs Repertoiretypen. Die Skalen reichen von 0 bis 100. Die Blasengrössen stellen den Anteil der Repertoiretypen an der Schweizer Wohnbevölkerung dar. Datengrundlage: Befragung 2016 mit $n=3477$ Befragten.

Lesebeispiel: Der Repertoiretyp der «Intensivnutzer» hat einen Wert von 61,7 bei der Güte der Newsnutzung und einen Wert von 71,3 bei der Intensität der Newsnutzung.
«Intensivnutzer» nehmen bei der Intensität der Newsnutzung den Spitzenrang ein. In Bezug auf die konsumierte, durchschnittliche Qualität liegen die «Intensivnutzer» auf dem zweiten Platz, knapp hinter den «Homeland Oriented». Letztere nutzen zwar anteilig die qualitativ besten Medien, doch die Nutzungsintensität (Nutzungshäufigkeit und Breite des genutzten Angebots) ist sehr gering. Noch geringer ist die Intensität der Newsnutzung bei den «News-Deprivierten», bei denen ausserdem die genutzte Qualität unterdurchschnittlich ist. Nur beim Typ «Old World Boulevard» ist die Güte der Newsnutzung noch etwas geringer. Im unteren Mittelfeld der qualitativen und quantitativen Newsnutzung befinden sich die «Global Surfer», während sich der Typ «Old World \& Online» im oberen Mittelfeld positioniert.

Die Qualitätsbilanzen werden durch die Kombination der beiden Dimensionen ermittelt. Sie werden multipliziert und wiederum auf eine Skala von 0 (minimale Qualitätsversorgung) bis 100 (maximale Qualitätsversorgung) transformiert (vgl. Darstellung 15). Der
Maximalwert ist dabei nur eine theoretische Grösse, die eine tägliche intensive Nutzung aller Medien zu Informationszwecken messen würde. Für andere Lebensinhalte bliebe dann keine Zeit mehr.

Die beste Qualitätsbilanz mit 43,3 Punkten weisen die «Intensivnutzer» auf, die sich viel Zeit nehmen, um sich in verschiedenen Medien von zumeist guter Qualität über Nachrichten zu informieren. Dabei fällt es für die Qualitätsbilanz weniger ins Gewicht, wenn unter den genutzten Angeboten auch minderwertige Nachrichten zu finden sind. Dieser Effekt wird bei weitem kompensiert durch das Übergewicht an qualitativ guten Medien, die regelmässig genutzt werden. Den Gegenpol zu den gut versorgten «Intensivnutzern» bilden die «News-Deprivierten». Sie können gerade einmal einen Viertel der Qualiätsbilanz der «Intensivnutzer» verbuchen. Mit 11,5 Punkten weisen sie die deutlich schlechteste Qualitätsbilanz aller Repertoiretypen auf. Die «News-Deprivierten» sind extrem unterversorgt mit News, sowohl in qualitativer wie in quantitativer Hinsicht. Newsmedien werden wenig ge- 


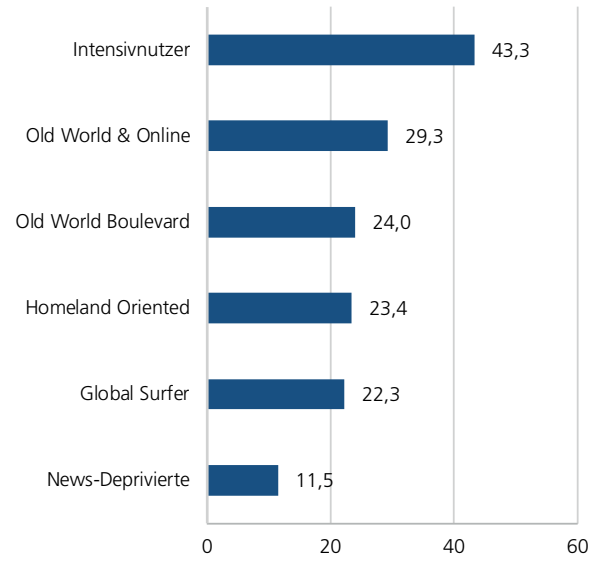

Darstellung 15: Repertoiretypen - Qualitätsbilanzen Die Darstellung zeigt die Qualitätsbilanzen für die sechs Repertoiretypen, die sich durch die Multiplikation der Repertoirequalität mit der Newsnutzungsquantität ergeben (Standardisierung der Skala auf 0 bis 100). Datengrundlage: Befragung 2016 mit $n=3477$ Befragten.

Lesebeispiel: Der Repertoiretyp der «News-Deprivierten» hat mit 11,5 Punkten die schlechteste Qualitätsbilanz aller Repertoiretypen.

nutzt, und wenn, dann vor allem solche von minderer Qualität. Auch der Repertoiretyp der «Global Surfer» schneidet in der Qualitätsbilanz mit 22,3 Punkten relativ schlecht ab. Er liegt sowohl bei der Qualität als auch bei der Quantität der Newsnutzung unter dem Durchschnitt und kann daher nicht an die Repertoiretypen heranreichen, die stärker in der traditionellen Newswelt beheimatet sind. Bei den «Global Surfern» drücken vor allem die eingeschränkte Medienauswahl und das selektive Nutzungsverhalten auf die Qualitätsbilanz.

Nach wie vor zeichnen sich die Newsrepertoires der «alten Medienwelt» vor allem durch traditionelle Medien aus, die eine überdurchschnittliche Güte aufweisen. Doch die Nutzungsfrequenz der traditionellen Medien, insbesondere der klassischen Tageszeitung, nimmt auch bei diesen Repertoiretypen immer mehr ab. Dieser Rückgang der quantitativen Newsnutzung wird (noch) nicht durch die Nutzung qualitativ hochwertiger Informationsangebote der «neuen Medienwelt» aufgefangen. Die Qualitätsbilanzen insbesondere der Typen «Old World Boulevard» und «Homeland Oriented» liegen deshalb mit 24,0 und 23,4 Punkten auf relativ niedrigem Niveau. Demgegenüber belegt der Repertoiretyp «Old World \& Online» den guten zweiten Platz im Bilanzranking. Aufgrund des breiten
Newsrepertoires, das qualitativ hochwertige Angebote sowohl der traditionellen als auch der neuen (Online-) Medienwelt abdeckt, liegt er mit 29,3 Punkten deutlich über dem Qualitätsdurchschnitt.

Die Repertoiretypen der «Intensivnutzer», "Global Surfer» und «News-Deprivierten» haben den Nutzungswandel von der «alten» zur «neuen Medienwelt» vollzogen. Allerdings sucht und findet nur die Minderheit der «Intensivnutzer» die qualitativ hochwertigen Newsangebote, die auch in der «neuen Medienwelt» vorhanden sind. Die Mehrheit - «Global Surfer» und insbesondere «News-Deprivierte» - nutzt hingegen qualitativ minderwertige Newsangebote. Traditionelle Qualitätsmedien sind in deren Newsrepertoires so gut wie nicht mehr vorhanden. Und neue Qualitätsangebote haben sie in ihre Newsrepertoires bislang nicht integriert.

\section{Soziodemografische Verortung der Repertoiretypen}

Anhand von soziodemografischen Variablen lassen sich die sozialen Kontexte der Repertoiretypen näher bestimmen. Diese Variablen der sozialen Lage geben Hinweise auf die Milieus, in denen die Nutzerinnen und Nutzer der jeweiligen Newsrepertoires verankert sind.

Die beiden Repertoiretypen «Homeland Oriented» und «News-Deprivierte» haben mit rund 60\% einen signifikant höheren Frauenanteil (vgl. Darstellung 16). Diese «weiblichen» Typen haben das geringste Newsinteresse. Demgegenüber ist der Gegenpol eindeutig männlich geprägt. Die «Intensivnutzer» mit ihrem breiten und qualitativ hochwertigen Newskonsum weisen mit gut 60\% ein klares Übergewicht an Männern auf. Die technikaffinen und international orientierten «Global Surfer» haben ebenfalls einen geringfügig höheren Anteil von Männern. Demgegenüber sind bei den beiden Repertoiretypen «Old World \& Online» und «Old World Boulevard», die noch stark in der traditionellen Newswelt verankert sind, die Geschlechteranteile ausgeglichen.

Im Hinblick auf das Alter zeigen sich deutliche Unterschiede zwischen den Repertoiretypen (vgl. Darstellung 17). Es ist daher durchaus zulässig, von den Newsrepertoires der jungen und der alten Nutzer zu sprechen. 


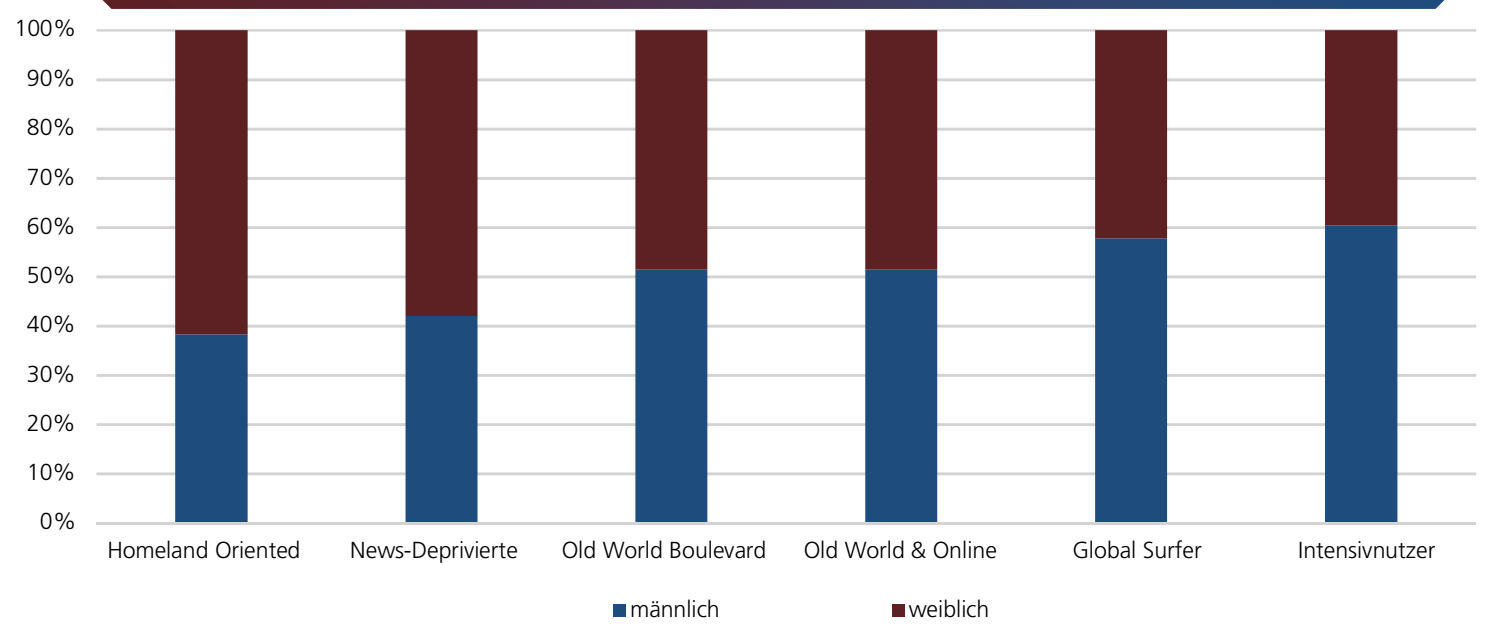

Darstellung 16: Repertoiretypen - Geschlechterprofile

Dargestellt sind die sechs Repertoiretypen sortiert von «stärker weiblich» (links) nach «stärker männlich» (rechts).

Datengrundlage: Erhebung 2016 mit $\mathrm{n}=3477$ Befragten.

Lesebeispiel: 62\% der Nutzerinnen und Nutzer des Repertoiretyps der «Homeland Oriented» sind Frauen, 38\% Männer.

jünger

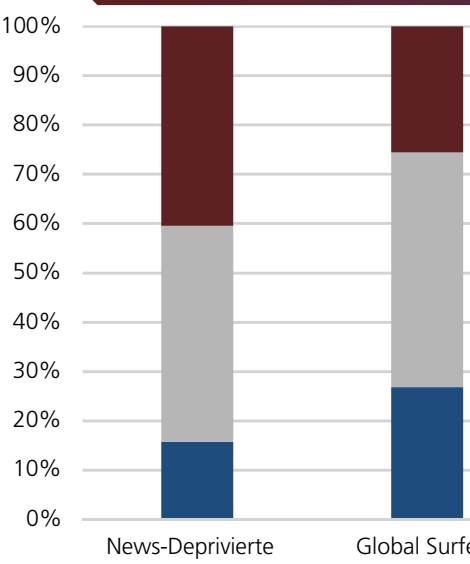

- 50-69 Jahre

- männlich

- weiblich 


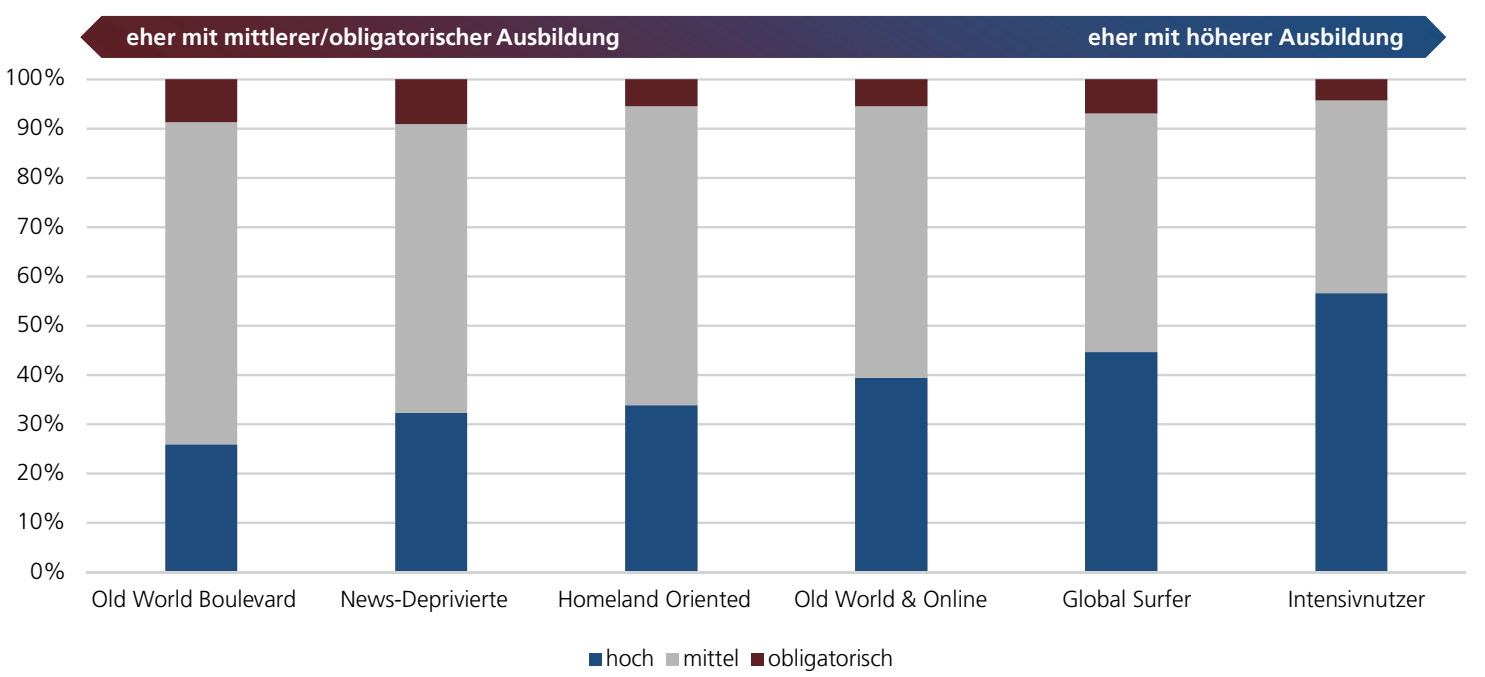

Darstellung 18: Repertoiretypen - Bildungsprofile

Dargestellt sind die sechs Repertoiretypen sortiert von «eher mit mittlerer/obligatorischer Ausbildung» (links) nach «eher mit höherer Ausbildung» (rechts). Datengrundlage: Erhebung 2016 mit $\mathrm{n}=3477$ Befragten.

Lesebeispiel: $26 \%$ der Nutzerinnen und Nutzer des Repertoiretyps der «Old World Boulevard» haben einen höheren Bildungsabschluss, $74 \%$ haben einen mittleren bzw. obligatorischen Bildungsabschluss.

insofern in dieser prägenden Phase keine vielfältige Newsnutzung stattfindet und gleichsam eine Gewöhnung an den diagnostizierten quantitativen und qualitativen Mangelzustand eintreten könnte. Dieser Befund gilt insbesondere für die unter 30 -jährigen Frauen, von denen fast die Hälfte zu den «News-Deprivierten» zählt (gegenüber einem Drittel der Männer in dieser Altersgruppe).

Ebenfalls ein junger Repertoiretyp sind die «Global Surfer». Auch sie sind kaum noch in die «alte Medienwelt» eingebunden und orientieren sich stark an den neuen Onlineangeboten. Doch dort nutzen sie bislang zu wenige Qualitätsangebote, um auf eine gute Qualitätsbilanz zu kommen.

Der älteste Repertoiretyp ist jener der «Homeland Oriented». Nur etwa $8 \%$ der Nutzerinnen und Nutzer in diesem Typ sind jünger als 30 Jahre, während die Hälfte 50 Jahre und älter ist. Die «Homeland Oriented» werden weiter an Bedeutung verlieren, ebenso wie der Typ «Old World \& Online». Diesem Repertoiretyp fehlt ebenfalls der «Nachwuchs», obwohl die Beimischung von Onlineangeboten ja durchaus modern und jugendlich anmutet. Es gibt aber schlicht zu wenig junge Nutzer, die in einer solchen Breite die traditionellen Newsmedien frequentieren. Dieser Repertoiretyp leidet somit weniger an Überalterung, sondern viel eher an «Unterjüngung», d.h. am mangelnden Nachwuchs aus den Reihen der jungen Alterskohorten.

Demgegenüber bleibt der Anteil der unter 30-Jährigen in den Repertoiretypen «Old World Boulevard» und «Intensivnutzer» mit jeweils gut 20\% stabil. Es scheint auch in der jungen Generation nennenswerte Anteile von Nutzerinnen und Nutzern zu geben, die von den Medienmachern mit ihrem gegenwärtigen Angebot angesprochen werden. Zum einen besteht Potenzial für die Rezeption von Boulevardjournalismus, zum anderen aber auch für hochwertigen Newsjournalismus. Dass der Repertoiretyp mit der besten Qualitätsbilanz, der durch sein breites Newsinteresse und seinen intensiven Newskonsum charakterisiert ist, vorerst nicht ausstirbt, sondern weiterhin junge «Intensivnutzer» nachwachsen, macht Hoffnung, dass auch in Zukunft hochwertiger Journalismus nachgefragt wird.

Mit Blick auf das soziodemografische Merkmal der Bildung zeigt sich, dass sich insbesondere im Repertoiretyp der «Intensivnutzer» die höheren Bildungsabschlüsse finden (vgl. Darstellung 18). Die intensive und qualitativ hochwertige Newsnutzung ist vor allem im bildungsbürgerlichen Milieu verankert. Dass Bildung nicht automatisch mit solchen quantitativ und qualitativ überdurchschnittlich versorgten Newsrepertoires verknüpft sein muss, zeigen allerdings die «Glo- 


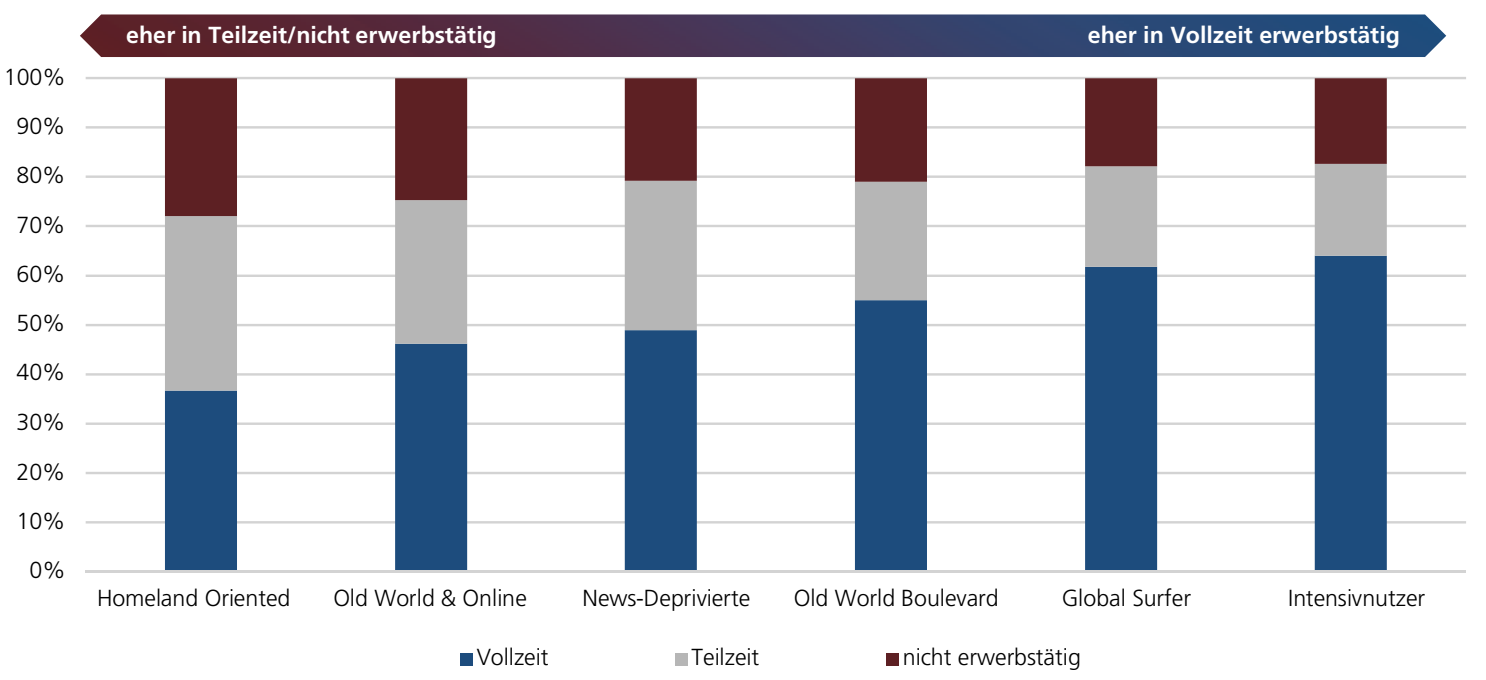

Darstellung 19: Repertoiretypen - Erwerbstätigkeitsprofile

Dargestellt sind die sechs Repertoiretypen sortiert von «eher in Teilzeit/nicht erwerbstätig» (links) nach «eher in Vollzeit erwerbstätig» (rechts). Datengrundlage: Erhebung 2016 mit $\mathrm{n}=3477$ Befragten.

Lesebeispiel: 53\% des Repertoiretyps der «Old World Boulevard» sind in Vollzeit erwerbstätig, 26\% in Teilzeit, 21\% sind nicht erwerbstätig.

bal Surfer». Obwohl dieser Typ ebenfalls einen überdurchschnittlichen Anteil von Personen mit höherer Bildung hat, bleiben deren Newsrepertoires im Hinblick auf die Qualitätsbilanz der genutzten News unter dem Durchschnitt.

Die unteren und mittleren Bildungsstufen sind vor allem bei den Typen «Old World Boulevard» und «News-Deprivierte» überrepräsentiert. Dabei ist zu bedenken, dass die «News-Deprivierten» zu einem grossen Anteil noch in Ausbildung sind und in Zukunft einen höheren Bildungsstatus erreichen werden. Es ist deshalb wichtig zu betonen, dass die Newsrepertoires der «News-Deprivierten» keineswegs automatisch mit geringer Bildung korrespondieren. Es ist bedenklich, dass viele junge Erwachsene kein Newsrepertoire ausprägen, das sie mit mehr und hochwertigeren News versorgt, obwohl sie die sekundäre und tertiäre Bildungsstufe absolviert haben oder absolvieren werden. Viele der gut ausgebildeten Digital Natives begnügen sich demnach mit News von minderer Qualität.

Die Repertoiretypen der «Intensivnutzer» und der «Global Surfer» weisen den höchsten Anteil von VollzeitErwerbstätigen auf (64\% und 62\%), gefolgt vom Typ «Old World Boulevard» mit 55\% (vgl. Darstellung 19). Während die «Intensivnutzer» und «Global Surfer» eher in hochqualifizierten Berufen tätig sind, überwiegen beim Typ «Old World Boulevard» die mittel und gering qualifizierten Berufstätigen. Der Anteil an nicht erwerbstätigen Mediennutzern ist bei den Repertoiretypen «Homeland Oriented» $(28 \%)$ und «Old World \& Online» (25\%) am höchsten, wobei die überwiegende Anzahl dieser nicht erwerbstätigen Personen pensioniert ist.

Weitere auffällige soziodemografische Verortungen der Repertoiretypen betreffen den Wohnort der Mediennutzer. Es zeigt sich, dass die stark an den neuen Newsmedien orientierten Repertoiretypen «Intensivnutzer», «Global Surfer» und «News-Deprivierte» in den grossen Städten und deren Einzugsgebieten deutlich übervertreten sind. Die medientechnischen Neuerungen setzen sich erwartungsgemäss in den urbanen Zentren durch und diffundieren dann weiter in die ländliche Peripherie. Wie wir gesehen haben, muss dies nicht automatisch mit einem Vorteil bezüglich der konsumierten, journalistischen Qualität einhergehen. In den ländlichen Kantonen sind die regionalen Abonnementszeitungen nach wie vor sehr stark in den Newsrepertoires verankert. Mit ihrer vergleichsweise hohen Qualität sorgen sie für ausgeglichene Qualitätsbilanzen auf dem Land. In den grossen Städten gleicht der höhere Anteil von «Intensivnutzern» die schlechteren Qualitätsbilanzen der anderen Newsrepertoires aus. In der Suisse romande kommen die Repertoiretypen «Intensivnutzer» und «Old World Boulevard» über- 


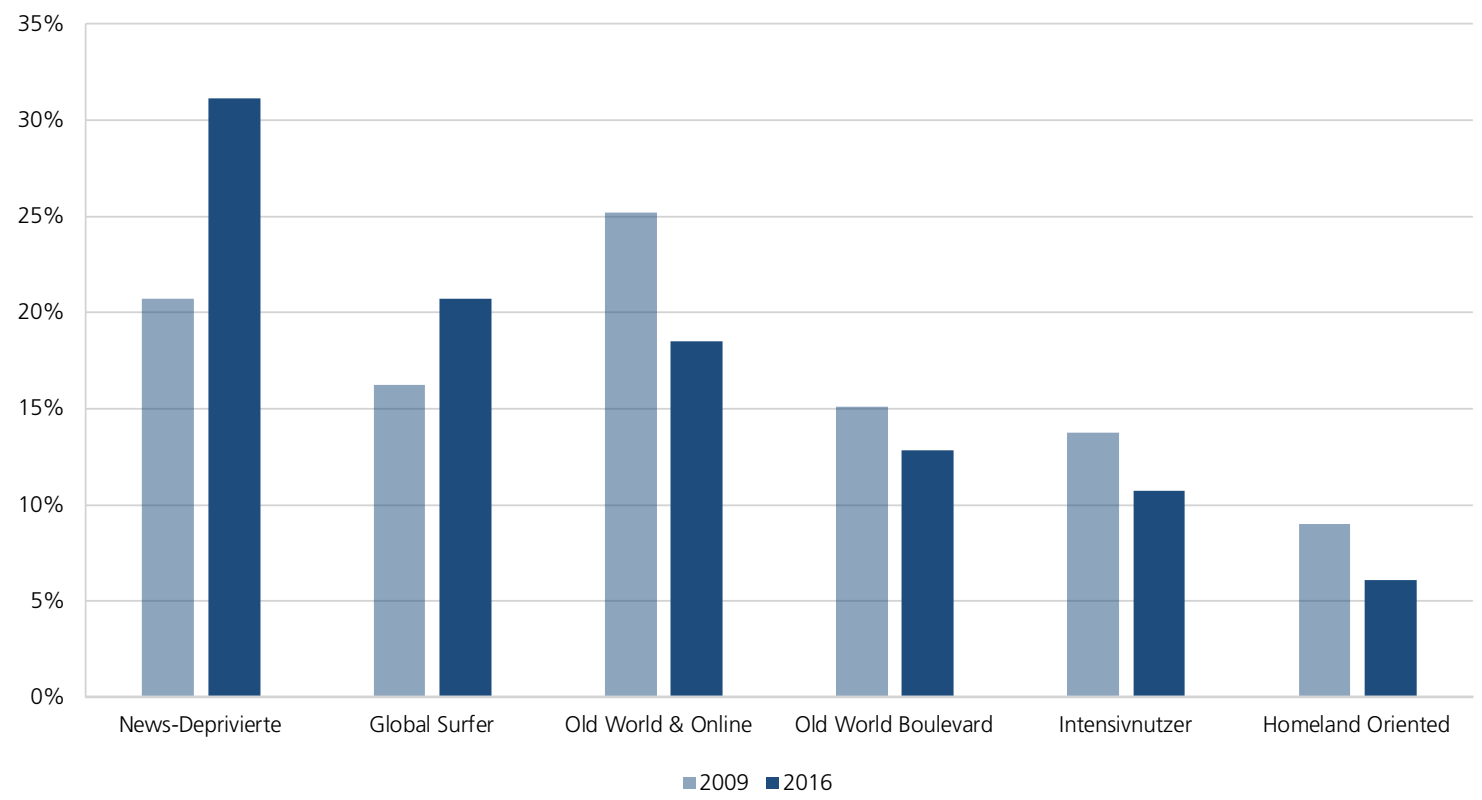

Darstellung 20: Repertoiretypen - Veränderung zwischen 2009 und 2016

Die Darstellung bildet den Anteil der Repertoiretypen an der Schweizer Wohnbevölkerung in den Jahren 2009 (hellblaue Säulen) und 2016 (dunkelblaue Säulen) ab. Geordnet sind die Repertoiretypen nach absteigenden Anteilswerten für das Jahr 2016. Datengrundlage: Erhebung 2009 mit $n=3331$ Befragten und Erhebung 2016 mit $\mathrm{n}=3477$ Befragten.

Lesebeispiel: Der Anteil der «News-Deprivierten» ist von 2009 bis 2016 um beträchtliche 10 Prozentpunkte auf heute $31 \%$ gestiegen.

proportional häufig vor, während in der Deutschschweiz die Typen «Homeland Oriented» und «Old World \& Online» übervertreten sind. In diesen Unterschieden zwischen den Sprachregionen kommen einerseits die stärkere Bedeutung der Boulevardpresse mit Le Matin und Le Matin Dimanche (mit ihren jeweiligen Onlineausgaben) in der Suisse romande und andererseits das stärkere Gewicht der Radionutzung in der Deutschschweiz zum Ausdruck.

Schliesslich sind auch im Hinblick auf die Nationalität der Nutzerinnen und Nutzer Unterschiede zwischen den Repertoiretypen zu beobachten. Einen erhöhten Anteil von Personen mit nichtschweizerischer Nationalität oder doppelter Staatsbürgerschaft haben die Typen der «Intensivnutzer» (fast 60\% höher als im Schnitt) und der «Global Surfer» (33\% höher als im Schnitt). Ein hoher Prozentsatz der Ausländer und Schweizer mit doppelter Staatsbürgerschaft ist als «Intensivnutzer» gut in die schweizerische Medienarena integriert. Sie nutzen das Medienangebot in seiner ganzen Breite und haben eine Qualitätsbilanz, die nach oben ausschwingt. Allerdings sind die Ausländer und Schweizer mit doppelter Staatsbürgerschaft auch im Repertoiretyp der "Global Surfer», der durch die Abwendung von den schweizerischen Medienangeboten charakterisiert ist, überproportional vertreten. Es zeigt sich, dass insbesondere die gut ausgebildeten Erwerbstätigen aus den westlichen Zentrumsnationen, die keine doppelte Staatsbürgerschaft besitzen und damit von der politischen Partizipation in der Schweiz ausgeschlossen sind, Newsrepertoires ausprägen, die der regionalen und nationalen Medienarena wenig Aufmerksamkeit widmen.

\section{Anteilsveränderungen der Repertoiretypen zwischen 2009 und 2016}

Mit über 31\% und einem satten Zuwachs von 10 Prozentpunkten seit 2009 sind die «News-Deprivierten» 2016 mittlerweile klar der grösste Repertoiretyp. Ebenso können die «Global Surfer» um starke 5 Prozentpunkte auf $21 \%$ zulegen und damit den zweiten Platz einnehmen (vgl. Darstellung 20). Zusammen vereinen diese beiden Typen mit ihren internetaffinen 
Newsrepertoires und ihren schlechten Qualitätsbilanzen inzwischen über die Hälfte der Mediennutzerinnen und -nutzer. In der rasanten Anteilssteigerung dieser Repertoiretypen spiegelt sich auf der Nutzungsseite, was auf der Angebotsseite als rasante Digitalisierung der Medien diagnostiziert und als Niedergang der gedruckten Abonnementspresse beklagt wird. Oder mit Blick auf die Fragen nach der Finanzierung und der Qualität des angebotenen Newsjournalismus formuliert: «News-Deprivierte» und «Global Surfer» sind «Kinder» des Booms von gratis verfügbaren Onlineund Printformaten, die zumeist qualitätsschwach sind. Der Anteil der Repertoiretypen mit den Repertoires der «alten Medienwelt» «Old World \& Online», «Old World Boulevard» und «Homeland Oriented» schrumpft seit 2009. Zusammengenommen verlieren diese drei Typen 12 Prozentpunkte. Am stärksten büsst der Typ «Old World \& Online» Anteile ein: 7 Prozentpunkte seit 2009 auf 18\% im Jahr 2016. Während «Old World \& Online» 2009 noch der grösste Repertoiretyp war, liegt er heute auf dem dritten Platz mit weiter sinkender Tendenz. Der wichtigste Grund für diese Verluste ist die angesprochene "Unterjüngung», im Sinne des fehlenden Nachwachsens von unter 30-Jährigen. Ausserdem sind gewisse Abwanderungen, insbesondere zu den «Global Surfern», zu beobachten, die mit der Abwendung einiger Nutzer von der traditionellen Zeitung hin zu den Onlineangeboten zusammenhängen.

Der Anteil der «Intensivnutzer» beträgt $11 \%$ und ist seit 2009 um 3 Prozentpunkte rückläufig. Gleichwohl lässt sich feststellen, dass der Anteil der «Intensivnutzer» seit 2012 stabil zwischen 10 und 11\% liegt. Der Abwärtstrend scheint demnach gebrochen zu sein. Einer von zehn Mediennutzern, dieser Sockel von Newsinteressierten, der vielfältige Medienangebote off- und online konsumiert und darüber hinaus bereit ist, für guten Journalismus zu bezahlen, darf nicht abbröckeln, damit Newsjournalismus auch in Zukunft finanziert werden kann.

\section{Themenagenden - welche Kommunikationsereignisse nehmen die Repertoiretypen wahr?}

Aus einer Liste von 20 berichterstattungsstarken Kommunikationsereignissen aus dem Jahr 2015 wählten die Befragten fünf gemäss der Vorgabe «Bitte geben Sie die 5 Ereignisse an, die Sie selbst am intensivsten verfolgt haben» aus. Die ausgewählten Kommunikationsereignisse sortierten die Befragten in Form einer Rangliste auf die Plätze 1 bis 5 (vgl. Darstellung 21).

Die Themenagenda der Schweizer für das Jahr 2015 wird von vier Kommunikationsereignissen dominiert: den Terroranschlägen in Paris, der Flüchtlingsproblematik, den Terroranschlägen des sogenannten Islamischen Staates und der Aufhebung des Euromindestkurses für den Schweizer Franken. Vier bis fünf von zehn Personen setzen jeweils diese Kommunikationsereignisse zuoberst auf ihre persönliche Agenda der Top-5Ereignisse 2015. Dieses Ranking lässt sich in groben Zügen bei allen sechs Repertoiretypen wiederfinden. Die grundlegende Integrationsleistung der Medien, für die Öffentlichkeit ein Set von Themen zu definieren, die für alle Mediennutzer eine gemeinsame Agenda bilden, wird demnach zumindest für die dominierenden Kommunikationsereignisse eines Jahres erbracht.

Im Schatten dieser Topthemen, die bei allen Mediennutzern eine gewisse Aufmerksamkeit erregen und das Publikum thematisch fokussieren, fragmentieren sich die Themenagenden jedoch zunehmend. Es zeigt sich, dass die Schwerpunktsetzungen innerhalb der Themenagenda sehr stark abweichen, je nachdem welches Newsrepertoire genutzt wird. Diese Schwerpunktsetzungen sind nicht allein mit soziodemografischen oder milieuspezifischen Unterschieden zu erklären. Der zentrale Erklärungsfaktor sind die unterschiedlichen Newsrepertoires selbst. Bestimmte Kommunikationsereignisse erhalten in bestimmten Medien mehr Resonanz und lenken damit die Aufmerksamkeit der Nutzerinnen und Nutzer auf diese Ereignisse und Themen. Indem wir die Abweichungen der Aufmerksamkeit für einzelne Kommunikationsereignisse der Repertoiretypen im Vergleich zum Durchschnitt aller Nutzerinnen und Nutzer anhand sogenannter standardisierter Residuen auswerten, lassen sich die repertoirespezifischen Wahrnehmungsprägungen aufdecken. 


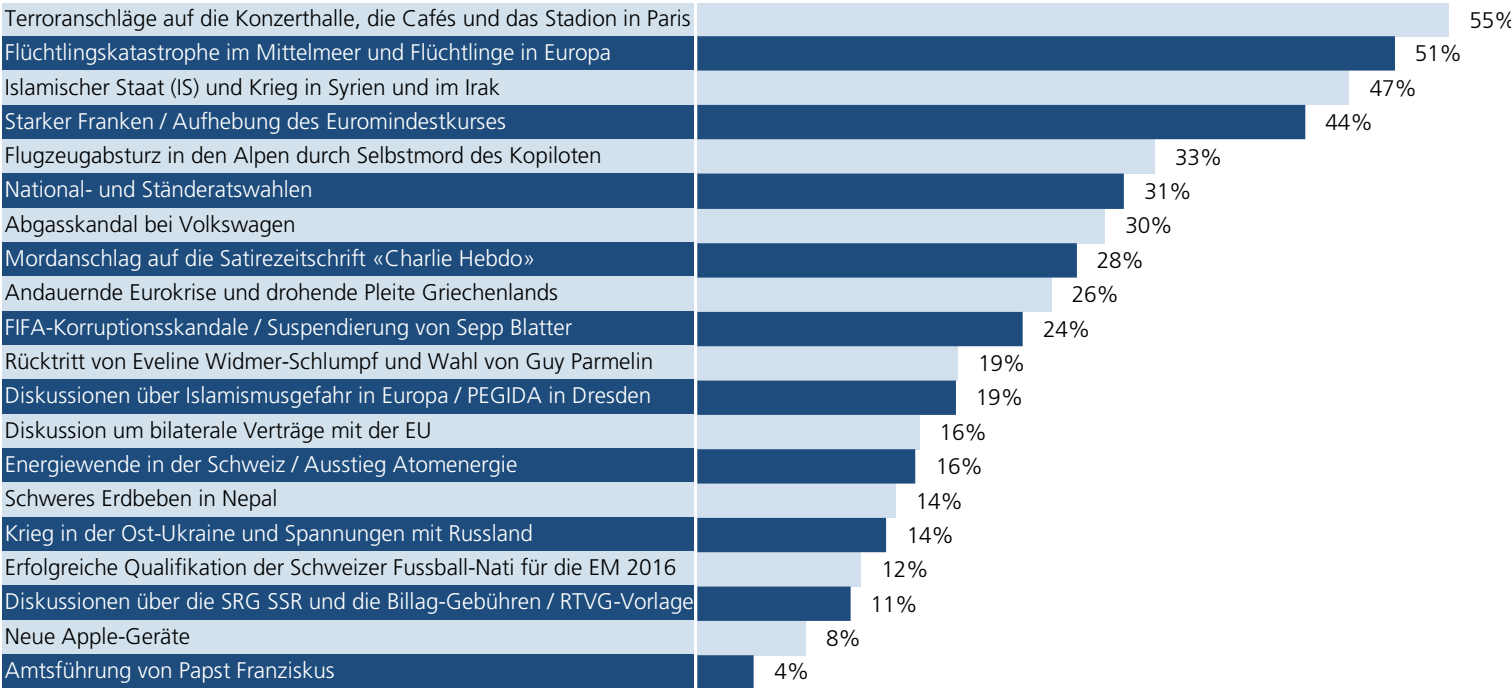

\section{Darstellung 21: Themenagenda 2015}

Die Darstellung zeigt den Anteil der Personen, die das Kommunikationsereignis auf einen der Top-5-Plätze der am intensivsten verfolgten Ereignisse im Jahr 2015 gesetzt haben. Datengrundlage: Erhebung 2016 mit $n=3477$ Befragten

Lesebeispiel: 54,5\% der Befragten bezeichnen die «Terroranschläge auf die Konzerthalle, die Cafés und das Stadion in Paris» als eines der fünf Ereignisse, die sie im Jahr 2015 am intensivsten verfolgt haben. Die «Amtsführung von Papst Franziskus» wurde dagegen nur von 4,1\% der Befragten als eines der Top-5-Ereignisse 2015 genannt.

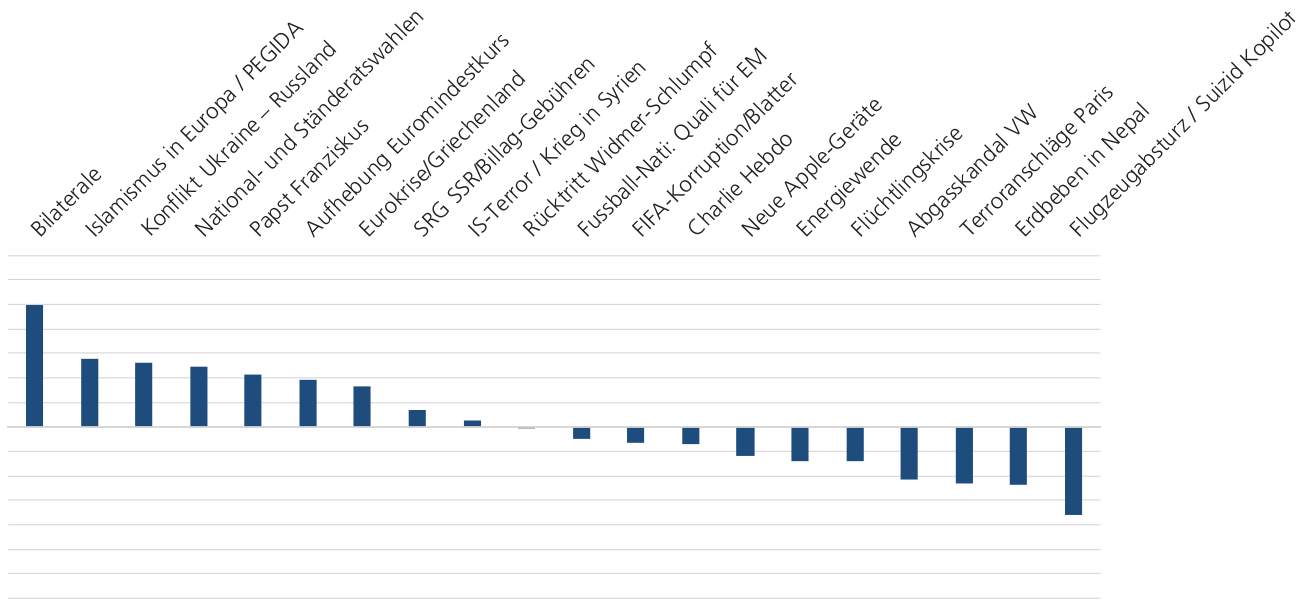

Darstellung 22: «Intensivnutzer» - Themenwahrnehmung

Dargestellt sind standardisierte Residuen, die angeben, wie über- bzw. unterdurchschnittlich ein Kommunikationsereignis vom Repertoiretyp im Vergleich zu allen Nutzerinnen und Nutzern wahrgenommen wird. Datengrundlage: Erhebung 2016 mit n = 373 Befragten des Repertoiretyps im Vergleich zu $\mathrm{n}=3477$ Befragten insgesamt.

Lesebeispiel: Die Aufmerksamkeit für die «Bilateralen» weicht in etwa doppelt so stark nach oben vom Durchschnitt aller Befragten ab wie die Aufmerksamkeit für die «National- und Ständeratswahlen». Die Aufmerksamkeit für den «Flugzeugabsturz / Suizid Kopilot» weicht am stärksten nach unten vom Durchschnitt aller Befragten ab.

Die Themenagenda des Repertoiretyps der «Intensivnutzer» ist stark durch relevante politische und wirtschaftliche Kommunikationsereignisse geprägt (vgl. Darstellung 22). Insbesondere die Aufmerksamkeit für die öffentlichen Diskussionen rund um die «Bilateralen» liegt weit über dem Durchschnitt. Internationale Vorgänge werden ebenfalls überdurchschnittlich intensiv verfolgt: «Islamismus in Europa / PEGIDA» und der 


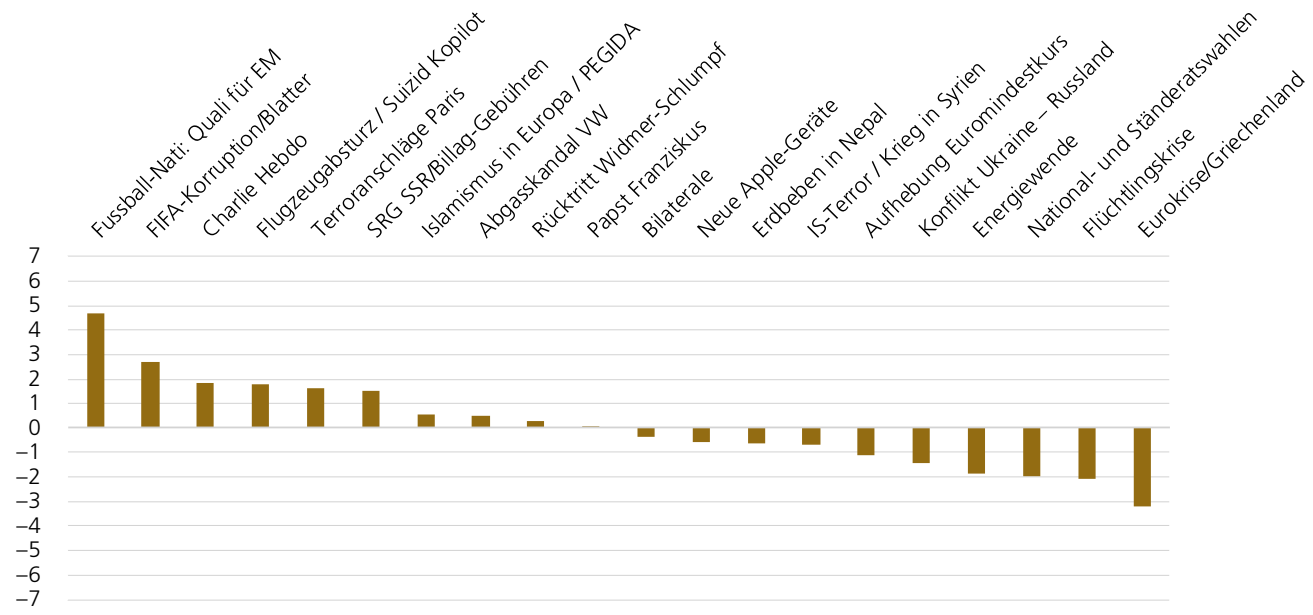

Darstellung 23: «Old World Boulevard» - Themenwahrnehmung

Dargestellt sind standardisierte Residuen, die angeben, wie über- bzw. unterdurchschnittlich ein Kommunikationsereignis vom Repertoiretyp im Vergleich zu allen Nutzerinnen und Nutzern wahrgenommen wird. Datengrundlage: Erhebung 2016 mit $n=446$ Befragten des Repertoiretyps im Vergleich zu $\mathrm{n}=3477$ Befragten insgesamt.

Lesebeispiel: Die Aufmerksamkeit für die «Fussball-Nati: Quali für EM» weicht in etwa doppelt so stark nach oben vom Durchschnitt aller Befragten ab wie die Aufmerksamkeit für die «FIFA-Korruption/Blatter». Die Aufmerksamkeit für den «Eurokrise/Griechenland» weicht am stärksten nach unten vom Durchschnitt aller Befragten ab.

«Konflikt Ukraine - Russland» sowie die «Amtsführung von Papst Franziskus» finden starke Beachtung Themen und Ereignisse, die auf der Agenda der breiten Bevölkerung auf hinteren Plätzen landeten. Weitere politische Kommunikationsereignisse mit zum Teil explizitem Wirtschaftsbezug sind die «National- und Ständeratswahlen», die «Aufhebung des Euromindestkurses», die «Eurokrise/Griechenland» und die medienpolitischen Diskussionen rund um die SRG SSR, u.a. im Zusammenhang der Abstimmung zur RTVG-Revision («SRG SSR/Billag-Gebühren»). Weniger stark verfolgt werden Kommunikationsereignisse rund um Krisen und Katastrophen.

Der Repertoiretyp «Old World Boulevard» rezipiert bevorzugt Sportthemen (vgl. Darstellung 23). Darüber hinaus ist seine Aufmerksamkeit auf Ereignisse von hoher Emotionalität gerichtet. Besonders emotionalisierend war die personenzentrierte Berichterstattung in den Boulevardmedien über die Terroranschläge in Frankreich und den Flugzeugabsturz der Fluggesellschaft Germanwings in den französischen Alpen, der durch den Suizid des Kopiloten ausgelöst wurde. Die Kontroverse um den Service-public-Auftrag der öffentlichen Rundfunkanstalt SRG SSR wurde vom Blick sehr stark bewirtschaftet, was sich ebenfalls in der
Themenagenda des Typs «Old World Boulevard» niederschlägt. Demgegenüber wurde das grosse internationale wirtschaftspolitische Thema des Jahres 2015, die «Eurokrise», in seiner Komplexität kaum vom Boulevard behandelt und spielt für Nutzerinnen und Nutzer der boulevardorientierten Newsrepertoires nur eine untergeordnete Rolle. Ebenso wurde das andere grosse internationale Kommunikationsereignis, die «Flüchtlingskatastrophe im Mittelmeer und Flüchtlinge in Europa», mit seiner humanitär-politischen Fokussierung von diesem Typ weniger wahrgenommen als das Thema des «Islamismus in Europa / PEGIDA», das den Blick eher auf die Bedrohungsproblematik der internationalen Entwicklungen lenkt.

Die Themenagenda des Repertoiretyps «Old World \& Online» wird ganz eindeutig von der schweizerischen Innenpolitik dominiert (vgl. Darstellung 24). Die Aufmerksamkeit für die «eidgenössischen National- und Ständeratswahlen» schwingt weit nach oben aus und sprengt geradezu die Skala. Ebenso werden der «Rücktritt von Eveline Widmer-Schlumpf und Wahl von Guy Parmelin» und die Diskussionen um die «Bilateralen» überdurchschnittlich intensiv verfolgt. In diesen Aufmerksamkeitsstrukturen bildet sich die Ausrichtung der Newsrepertoires ab, die bei diesem Typ vor allem 


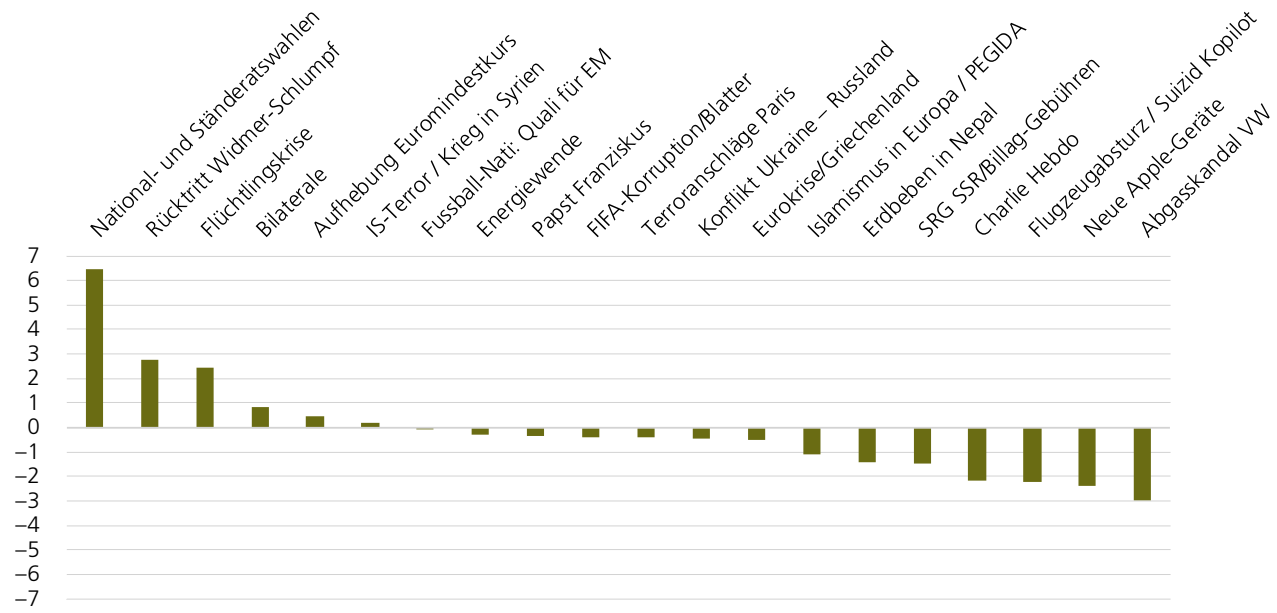

\section{Darstellung 24: "Old World \& Online" - Themenwahrnehmung}

Dargestellt sind standardisierte Residuen, die angeben, wie über- bzw. unterdurchschnittlich ein Kommunikationsereignis vom Repertoiretyp im Vergleich zu allen Nutzerinnen und Nutzern wahrgenommen wird. Datengrundlage: Erhebung 2016 mit $n=643$ Befragten des Repertoiretyps im Vergleich zu $\mathrm{n}=3477$ Befragten insgesamt.

Lesebeispiel: Die Aufmerksamkeit für die «National- und Ständeratswahlen» weicht rund dreimal so stark nach oben vom Durchschnitt aller Befragten ab wie die Aufmerksamkeit für den «Rücktritt von Eveline Widmer-Schlupf und die Wahl von Guy Parmelin». Die Aufmerksamkeit für den «Abgasskandal bei VW» weicht am stärksten nach unten vom Durchschnitt aller Befragten ab.

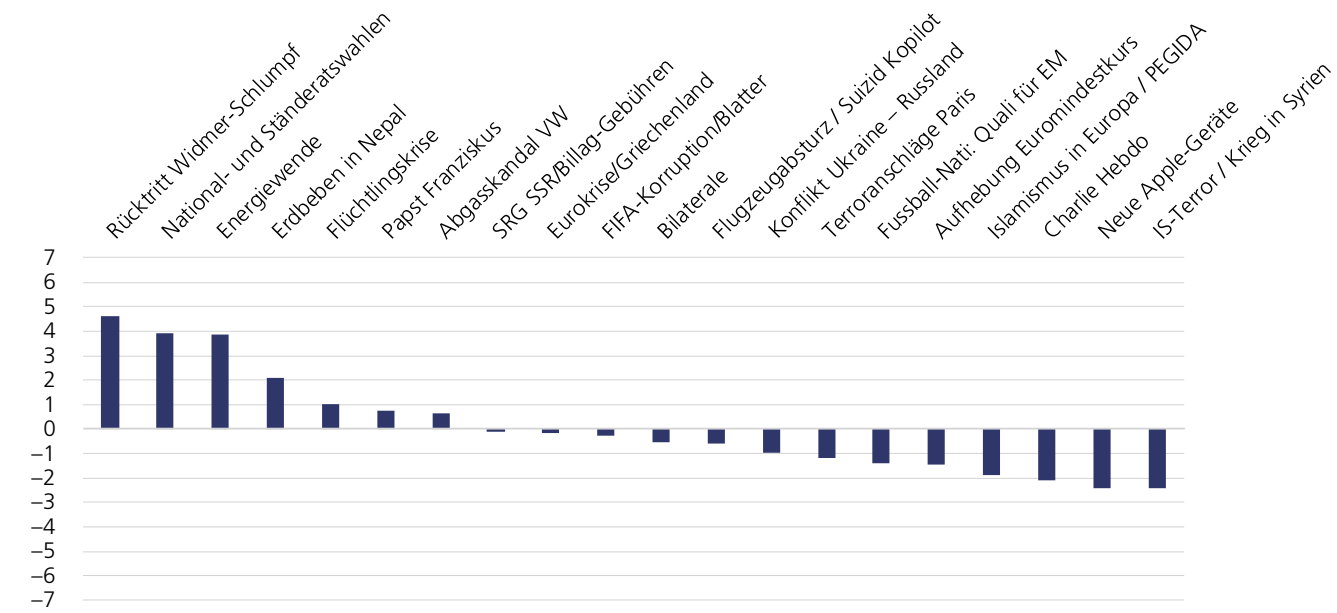

Darstellung 25: «Homeland Oriented» - Themenwahrnehmung

Dargestellt sind standardisierte Residuen, die angeben, wie über- bzw. unterdurchschnittlich ein Kommunikationsereignis vom Repertoiretyp im Vergleich zu allen Nutzerinnen und Nutzern wahrgenommen wird. Datengrundlage: Erhebung 2016 mit $n=212$ Befragten des Repertoiretyps im Vergleich zu $\mathrm{n}=3477$ Befragten insgesamt.

Lesebeispiel: Die Aufmerksamkeiten für den «Rücktritt von Eveline Widmer-Schlumpf und die Wahl von Guy Parmelin», die «National- und Ständeratswahlen» und die «Energiewende» weichen in etwa gleich stark nach oben vom Durchschnitt aller Befragten ab. Die Aufmerksamkeit für den «Islamischen Staat (IS) und den Krieg in Syrien und im Irak» weicht am stärksten nach unten vom Durchschnitt aller Befragten ab.

die nationale Medienarena abdecken und stark auf Politikthemen fokussieren. Unternehmensbezogene Kommunikationsereignisse landen bei diesem Repertoiretyp auf den hinteren Rängen der Themenagenda. Das gilt sowohl für Kommunikationsereignisse mit überwiegend positiver Resonanz wie bei der PR-lastigen Berichterstattung über «Neue Apple-Geräte» als auch für Ereignisse mit sehr negativer Resonanz wie die Berichterstattung über den «Abgasskandal bei VW». 


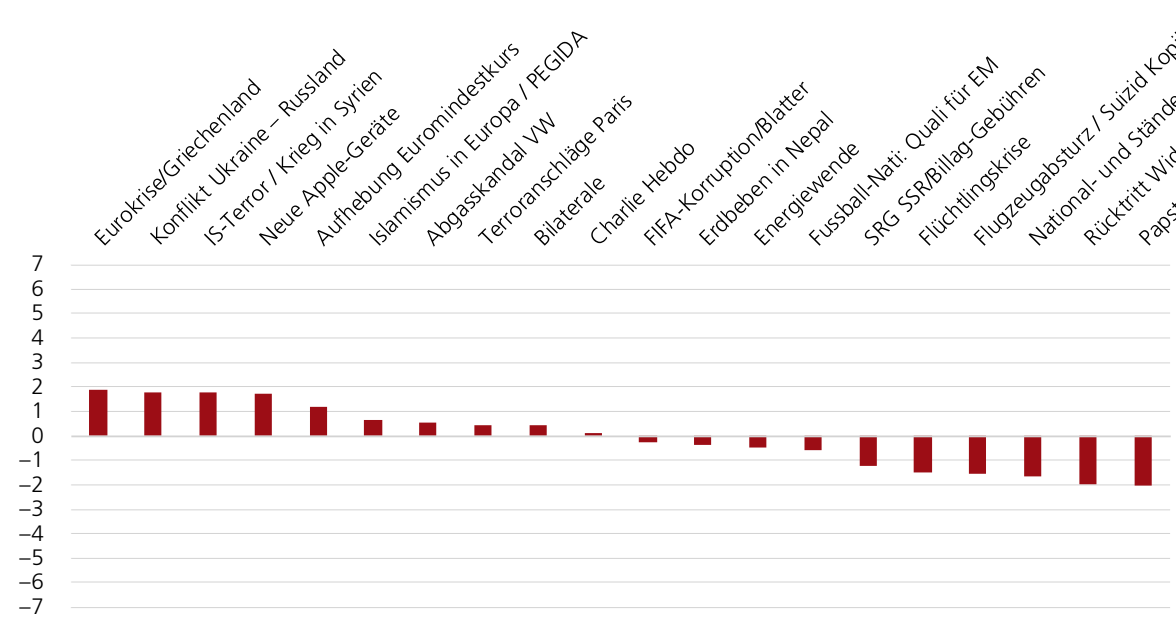

Darstellung 26: «Global Surfer» - Themenwahrnehmung

Dargestellt sind standardisierte Residuen, die angeben, wie über- bzw. unterdurchschnittlich ein Kommunikationsereignis vom Repertoiretyp im Vergleich zu allen Nutzerinnen und Nutzern wahrgenommen wird. Datengrundlage: Erhebung 2016 mit $n=721$ Befragten des Repertoiretyps im Vergleich zu $\mathrm{n}=3477$ Befragten insgesamt.

Lesebeispiel: Die Aufmerksamkeiten der «Global Surfer» weichen relativ wenig vom Durchschnitt aller Befragten ab. Die stärkste Abweichung nach oben ist für die «Eurokrise/Griechenland» zu beobachten. Die Aufmerksamkeiten für den «Rücktritt von Eveline Widmer-Schlumpf und die Wahl von Guy Parmelin» und «Amtsführung von Papst Franziskus» weichen am stärksten nach unten vom Durchschnitt aller Befragten ab.

Der Repertoiretyp «Homeland Oriented» rechtfertigt seine Bezeichnung durch seine Themenagenda (vgl. Darstellung 25). Ebenso wie beim Typ «Old World \& Online» stehen eindeutig innenpolitische Kommunikationsereignisse auf den vorderen Plätzen der Themenagenda: «Rücktritt Widmer-Schlumpf», «National- und Ständeratswahlen» und die «Energiewende» - ein inhaltlich komplexes und kommunikativ schwer zu vermittelndes Thema, das vor allem durch regionale Betroffenheit eine gewisse Brisanz erhält. Der eher auf Sicherheit und Harmonie bedachte Repertoiretyp «Homeland Oriented» verbannt Kommunikationsereignisse mit ausgeprägten Bedrohungsszenarien rund um Krieg, Terror und Fundamentalismus auf die hinteren Plätze seiner Themenagenda. Auch Wirtschaftsthemen erhalten von diesem Typ deutlich weniger Aufmerksamkeit als von Nutzern der anderen Newsrepertoires. Dass «Neue Apple-Geräte» abgeschlagen auf dem zweitletzten Platz landen, erstaunt angesichts der geringen Technikaffinität der «Homeland Oriented» nicht.

Die Agenden der «Global Surfer» in Bezug auf die von ihnen intensiv verfolgten Kommunikationsereignisse sind äusserst disparat. Es gibt kaum einzelne Kommunikationsereignisse, die im Vergleich mit der Agenda der breiten Bevölkerung besonders hervorstechen (vgl. Darstellung 26).

Besonders auffällig ist die fehlende Wahrnehmung der regionalen und nationalen Themen. Nur wenn nationale Themen Beziehungen zum Ausland betreffen und wirtschaftlich relevant sind («Aufhebung Euromindestkurs» und «Bilaterale»), finden diese bei den «Global Surfern» eine gewisse Aufmerksamkeit. Ansonsten ziehen die krisenhaften internationalen Politikthemen «Eurokrise/Griechenland», «Konflikt Ukraine - Russland» und «IS-Terror / Krieg in Syrien» und unternehmensbezogene Kommunikationsereignisse («Neue Apple-Geräte», «Abgasskandal VW») etwas gesteigerte Aufmerksamkeit auf sich. Bei diesem Repertoiretyp findet ein Rückzug aus der regionalen und schweizerischen Themenagenda statt zugunsten einer globalen Perspektive. Diese gut ausgebildeten Berufstätigen, von denen viele einen Migrationshintergrund aus westlichen Zentrumsnationen haben, nehmen an den gesellschaftspolitischen Debatten der Schweiz kaum Anteil. Es ist bedenklich, dass sich diese Personen grösstenteils aus der nationalen Öffentlichkeit «ausklinken». Es ist anzunehmen, dass wohl vor allem die mangelnden politischen Partizipationsmöglichkeiten für dieses Desinteresse verantwortlich sind. 


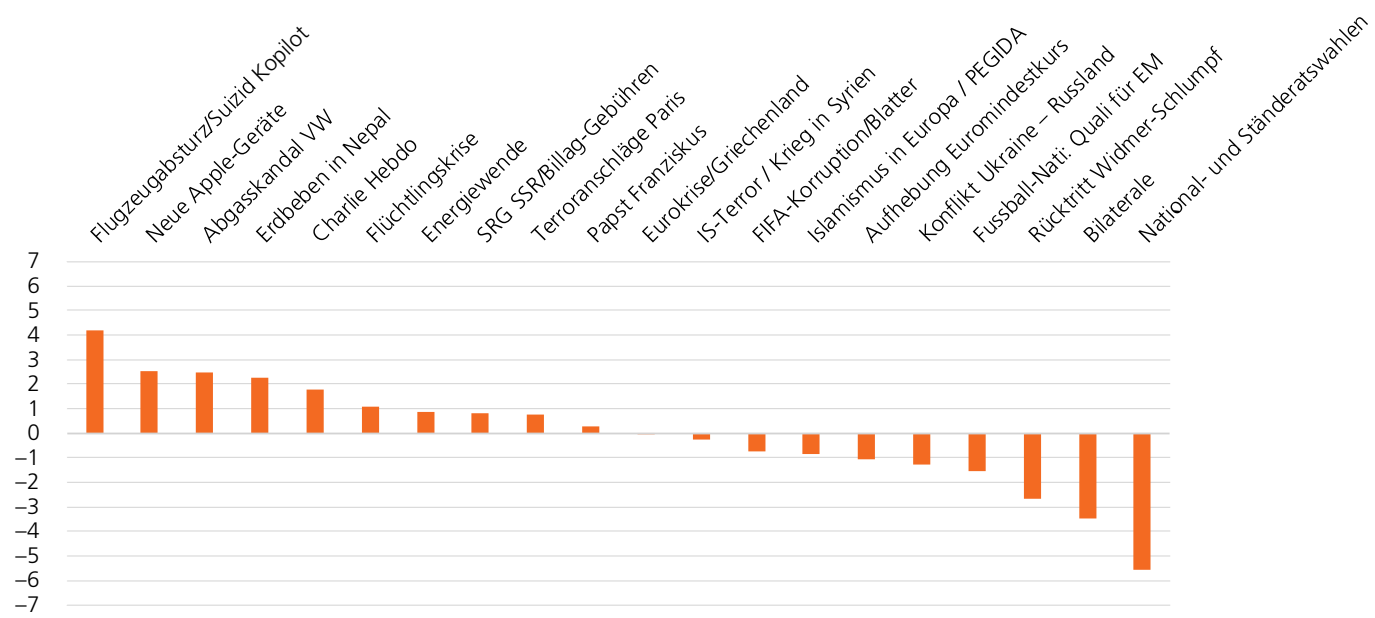

Darstellung 27: «News-Deprivierte» - Themenwahrnehmung

Dargestellt sind standardisierte Residuen, die angeben, wie über- bzw. unterdurchschnittlich ein Kommunikationsereignis vom Repertoiretyp im Vergleich zu allen Nutzerinnen und Nutzern wahrgenommen wird. Datengrundlage: Erhebung 2016 mit $n=1083$ Befragten des Repertoiretyps im Vergleich zu $\mathrm{n}=3477$ Befragten insgesamt.

Lesebeispiel: Die Aufmerksamkeit für den «Flugzeugabsturz / Suizid des Kopiloten» weicht in etwa 1,5-mal so stark nach oben vom Durchschnitt aller Befragten ab wie die Aufmerksamkeit für «Neue Apple-Geräte». Die Aufmerksamkeit für die «National- und Ständeratswahlen» weicht am stärksten nach unten vom Durchschnitt aller Befragten ab.

Der Repertoiretyp der «News-Deprivierten» ist zwar oberflächlich über die resonanzstarken Kommunikationsereignisse des Jahres 2015 informiert. Bei einem differenzierten Blick fallen aber starke Abweichungen von den Themenagenden der anderen Typen auf: Die quantitativ wie qualitativ abfallenden Newsrepertoires der «News-Deprivierten» mit ihren extrem schlechten Qualitätsbilanzen führen dazu, dass Einzelereignisse statt Themenzusammenhänge, personalisierte Storys statt komplexe Entwicklungen und Softnews statt Hardnews die stärkste Aufmerksamkeit erhalten (vgl. Darstellung 27).

Die Themenagenden der «News-Deprivierten» sind viel mehr durch Katastrophen («Flugzeugabsturz / Suizid Kopilot», «Erdbeben in Nepal»), Krisen («Flüchtlinge») und Terror («Charlie Hebdo», «Terroranschläge in Paris») geprägt als die der Nutzerinnen und Nutzern anderer Newsrepertoires. Wirtschaft wird vor allem anhand übertrieben positiver Hypes («Neue Apple-Geräte») oder extrem negativer Skandalberichterstattung («Abgasskandal VW») wahrgenommen. Komplexere wirtschaftliche und wirtschaftspolitische Themen, wie die «Aufhebung des Euromindestkurses» sowie die «Eurokrise/Griechenland», gehen dagegen unter. Die Kommunikationsereignisse der schweizeri- schen Tagespolitik stehen am Ende der Themenagenda. Insbesondere die «National- und Ständeratswahlen» wurden von den «News-Deprivierten» deutlich seltener verfolgt als von allen anderen Repertoiretypen.

Bedrohliche Ereignisse, oftmals partikulär und journalistisch kaum eingeordnet, stehen im Fokus der «NewsDeprivierten». Dieser von Bedrohungsbildern geprägte Blick auf die Gesellschaft und die Welt, verbunden mit der geradezu systematischen Ausblendung politischer Prozesse und Zusammenhänge, macht die «NewsDeprivierten» in besonderem Masse anfällig für populistische Angstpolitik mit ihren scheinbar einfachen Lösungen.

\section{Fazit}

Indem wir die Mediennutzung durch Repertoires beschreiben und in die Lebenswelt der Nutzerinnen und Nutzer einbetten, erhalten wir ein leistungsstarkes Analyseinstrument, um Effekte der Mediennutzung aufdecken und erklären zu können. Der Vorteil der Repertoireforschung besteht darin, dass wir nicht von einzelnen Medien ausgehen und deren Nutzung isoliert betrachten. Vielmehr setzen wir beim einzelnen Mediennutzer an und nehmen mit dem Repertoirean- 
satz das gesamte Spektrum der Medien in den Blick, die dieser Mediennutzer verwendet, um sich zu informieren. Bezogen auf das Informationsverhalten lassen sich so Newsrepertoires als individuell unterschiedliche Kombinationen und Nutzungsintensitäten von verschiedenen Medientypen und Medientiteln identifizieren. Ähnliche Newsrepertoires werden zu Repertoiretypen zusammengefasst, die nach innen sehr homogen sind und sich nach aussen klar voneinander abgrenzen. Die Repertoiretypen werden soziodemografisch und milieuspezifisch verortet, sodass die Logik der Newsnutzung nachvollziehbar wird. Ausserdem können wir durch die Kombination mit der inhaltsanalytischen Medienqualitätsforschung für die einzelnen Newsrepertoires Qualitätsbilanzen erstellen, die für die Repertoiretypen aggregiert werden und deren quantitative und qualitative Versorgung mit News zeigen. So gewinnen wir einen weiteren Erklärungsfaktor, um Mediennutzungseffekte anhand der Repertoiretypen zu analysieren.

Empirisch lassen sich sechs stabile Repertoiretypen bilden und in ihrer Entwicklung über die letzten acht Jahre beschreiben. Stark in der traditionellen Medienwelt verhaftet sind die Repertoiretypen «Homeland Oriented», «Old World Boulevard» und «Old World \& Online», wobei der letztgenannte Typ die grösste Öffnung hin zur neuen, digitalisierten Medienwelt aufweist. In beiden Welten verankert ist der Repertoiretyp der «Intensivnutzer», der sowohl traditionelle als auch online vermittelte Newsangebote ausgiebig in Anspruch nimmt. Am stärksten in der neuen Medienwelt beheimatet sind die Repertoiretypen der «Global Surfer» und der «News-Deprivierten». Es zeigt sich, dass in den letzten Jahren die beiden Typen «Global Surfer» und «News-Deprivierte» einen massiven Zuwachs verzeichnen und inzwischen die Mehrheit der Nutzerinnen und Nutzer repräsentieren. Dies ist einerseits als Ergebnis der fortschreitenden Digitalisierung der Medien ein Zeichen der Modernisierung der Mediennutzung. Andererseits gibt der Befund, dass gerade diese beiden Repertoiretypen die schlechtesten Qualitätsbilanzen in Bezug auf den konsumierten Newsjournalismus haben, Anlass zur Sorge.

Die empirische Relevanz der Repertoiretypen erweist sich durch ihre Erklärungskraft für konkrete Mediennutzungseffekte. Signifikante Unterschiede in den Themenagenden der Nutzer können mithilfe der Re- pertoiretypen diagnostiziert werden. Darüber hinaus lassen sich diese Unterschiede in den Aufmerksamkeitsstrukturen der Mediennutzer plausibel erklären, indem sie aus den verschiedenen Newsrepertoires und den dahinterstehenden Lebenswelten eindeutig hergeleitet werden.

\section{Repertoiretypen und ihre Themenagenden}

Es gibt klare Fokussierungen bei den Themenagenden, die sich mit den Newsrepertoires erklären lassen:

Für den Repertoiretyp «Intensivnutzer» - er ist bildungsbürgerlich, eher männlich, oft mit Migrationshintergrund - ist es ein statushebendes Differenzierungsmerkmal, viel Zeit darauf zu verwenden, sich breit und vertiefend über News zu informieren und dabei insbesondere Qualitätsangebote zu nutzen. Seine Themenagenden sind durch gesellschaftspolitische und wirtschaftliche Relevanz gekennzeichnet.

Der Typ der «Old World Boulevard» - er gehört tendenziell zur unteren Mittelschicht und ist berufstätig nutzt vor allem traditionelle Medien, und insbesondere die Boulevardzeitung, um sich zu informieren. Auf seiner Agenda stehen Sport- und Human-InterestEreignisse, über die emotional und personenbezogen berichtet wird.

Der Repertoiretyp der «Old World \& Online» - er ist gebildet und interessiert - ergänzt sein traditionelles Set an Newsmedien mit ausgewählten Angeboten aus dem Internet. Für ihn als beflissenen Staatsbürger einer demokratischen Gesellschaft wie der Schweiz stehen die nationalen Politikthemen zuoberst auf der Agenda.

Der Typ der «Homeland Oriented» - er ist tendenziell älter, weiblich und häuslich - stützt sich auf die bewährten traditionellen Medien, die eher nebenbei als mit fokussiertem Newsinteresse genutzt werden. Die Agenden sind auf regionale und nationale Themen fokussiert. Bedrohliche Ereignisse und Themen werden gemieden.

Der Repertoiretyp der "Global Surfer» - er ist gut ausgebildet, berufstätig und technikaffin, oft mit Migrationshintergrund - nutzt vor allem das Internet als zentrale Informationsquelle. Er hat eine international ausgerichtete Agenda, aus der schweizerischen Medienarena zieht er sich zurück.

Der Repertoiretyp der «News-Deprivierten» - eher jung und eher weiblich - ist mit News quantitativ und qualitativ unterversorgt. Seine Wahrnehmung der Welt bleibt an der Oberfläche. Einzelereignisse und Bedro- 
hungsszenarien von Katastrophen, Krisen und Terror prägen seine Themenagenda. Politische Prozesse und Zusammenhänge bleiben ihm fremd.

\section{Literatur}

Bonfadelli, H., 2002: The Internet and Knowledge Gaps. A Theoretical and Empirical Investigation, in: European Journal of Communication, 17(1), 65-84.

fög - Forschungsinstitut Öffentlichkeit und Gesellschaft / Universität Zürich (Hg.), 2016: Jahrbuch 2016 Qualität der Medien. Schweiz - Suisse - Svizzera, Basel: Schwabe.

Franck, Georg, 2007: Ökonomie der Aufmerksamkeit. Ein Entwurf. München: dtv.

Hasebrink, Uwe 2008: Das multiple Publikum, in: Paradoxien des Journalismus. Theorie - Empirie - Praxis. Festschrift für Siegfried Weischenberg, hg. von Bernhard Pörksen / Wiebke Loosen / Armin Scholl / Siegfried Weischenberg. 1. Aufl. Wiesbaden: VS Verlag für Sozialwissenschaften, S. 513-530.

Hasebrink, Uwe / Popp, Jutta, 2006: Media repertoires as a result of selective media use. A conceptual approach to the analysis of patterns of exposure, in: Communications, 31(3).

Ksiazek, Thomas B. / Malthouse, Edward / Webster, James, 2010: News-seekers and Avoiders: Exploring Patterns of Total News Consumption Across Media and the Relationship to Civic Participation, in: Journal of broadcasting \& electronic media, 54(4), 551-568.

Levendusky, M. S., 2013: Why Do Partisan Media Polarize Viewers?, in: American Journal of Political Science, 57(3), 611-623.

Luhmann, Niklas, 2009: Die Realität der Massenmedien (Neue Bibliothek der Sozialwissenschaften). 4. Aufl. Wiesbaden: VS Verlag für Sozialwissenschaften.

Pariser, Eli, 2011: The filter bubble. What the Internet is hiding from you. New York, NY: Penguin Press.
Rössler, Patrick, 1997: Agenda-Setting. Theoretische Annahmen und empirische Evidenzen einer Medienwirkungshypothese (Studien zur Kommunikationswissenschaft, 27). Wiesbaden: VS Verlag für Sozialwissenschaften.

Strömbäck, Jesper / Shehata, Adam, 2010: Media malaise or a virtuous circle? Exploring the causal relationships between news media exposure, political news attention and political interest, in: European Journal of Political Research, 49(5), 575597.

Stroud, Natalie Jomini, 2011: Niche news. The politics of news choice. New York: Oxford University Press.

Stroud, Natalie Jomini, 2010: Polarization and Partisan Selective Exposure, in: Journal of Communication, 60, 556-576.

Webster, James G., 2014: The marketplace of attention. How audiences take shape in a digital age. Cambridge, Mass. u.a.: MIT Press.

Weiß, Ralph, 2010: «Typisch!» - Mediennutzung im Alltag. Die Mediennutzertypologie als Instrument der Gesellschaftsdiagnose, in: Die MedienNutzerTypologie 2.0. Aktualisierung und Weiterentwicklung des Analyseinstruments, hg. von Ekkehardt Oehmichen / Christa-Maria Ridder (Schriftenreihe Media-Perspektiven, 20). 1. Aufl. Baden-Baden: Nomos-Verl.Ges., S. 57-73.

Weiß, Ralph 2009a: Pierre Bourdieu: Habitus und Alltagshandeln, in: Schlüsselwerke der Cultural Studies, hg. von Andreas Hepp / Friedrich Krotz / Tanja Thomas (Medien, Kultur, Kommunikation). 1. Aufl. Wiesbaden: VS Verlag für Sozialwissenschaften, S. 31-46.

Weiß, Ralph, 2009b: Politisch-kommunikative Milieus. Notwendigkeit und Nutzen einer milieutheoretischen Analyse politischer Kommunikation, in: M\&K, 3-22. 


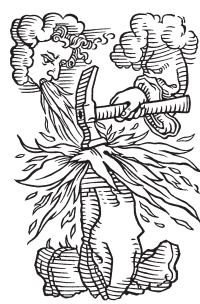

Das Signet des 1488 gegründeten Druck- und Verlagshauses Schwabe reicht zurück in die Anfänge der Buchdruckerkunst und stammt aus dem Umkreis von Hans Holbein. Es ist die Druckermarke der Petri; sie illustriert die Bibelstelle Jeremia 23,29: «Ist nicht mein Wort wie Feuer, spricht der Herr, und wie ein Hammer, der Felsen zerschmettert?» 


\section{Studien Qualität der Medien \\ Schweiz - Suisse - Svizzera}

Herausgegeben vom fög - Forschungsinstitut Öffentlichkeit und Gesellschaft / Universität Zürich im Auftrag der Kurt Imhof Stiftung für Medienqualität, Zürich. ISSN 2296-5114.

Die Studien von 2010 bis 2012 finden sich auch im Jahrbuch Qualität der Medien des jeweiligen Jahrgangs.

Mario Schranz, Jörg Schneider, Mark Eisenegger: Medienvertrauen - eine vergleichende Perspektive. SQM 1/2016. Ca. 15 Seiten. PDF E-Book. ISBN 978-3-7965-3652-6. Ca. sFr. 12.- / € (D) 12.- / € (A) 12.50.

Jörg Schneider, Mark Eisenegger: Wie Mediennutzer in die Welt schauen: Die Newsrepertoires der Schweizerinnen und Schweizer und ihre Themenagenden. SQM 2/2016. Ca. 25 Seiten. PDF E-Book. ISBN 978-3-7965-3653-3. Ca. sFr. 12.- / € (D) 12.- / € (A) 12.50 .

Jens Lucht, Linards Udris: Kommerzialisierung und Konzentration der Medien im internationalen Vergleich. SQM 1/2013.32 Seiten. PDF E-Book. ISBN 978-3-7965-3231-3. Ca. sFr. 12.- / € (D) 12.- / € (A) 12.50.

Mark Eisenegger, Joël Orizet, Mario Schranz: Medien im Social Web - Journalistischer Mehrwert? SQM 2/2013. 19 Seiten. PDF E-Book. ISBN 978-3-7965-3232-0. Ca. sFr. 12.- / € (D) 12.- / € (A) 12.50.

Linards Udris: Schweizer Medien im Wahlkampf. Qualität der Medienberichterstattung vor den Eidgenössischen Wahlen 2011. SQM 1/2012. 25 Seiten. PDF E-Book. ISBN 978-3-7965-3227-6. Ca. sFr. 12.- / € (D) 12.- / € (A) 12.50.

Mario Schranz, Mark Eisenegger: Onlinenews - Die Qualität von Presse- und Onlinetiteln im Direktvergleich. SQM 2/2012. 18 Seiten. PDF E-Book. ISBN 978-3-7965-3228-3. Ca. sFr. 12.- / € (D) 12.- / € (A) 12.50.

Mark Eisenegger, Patrik Ettinger: Kriminalitätsberichterstattung in der Schweizer Presse. SQM 3/2012. 31 Seiten. PDF E-Book. ISBN 978-3-7965-3229-0. Ca. sFr. 12.- / € (D) 12.- / € (A) 12.50.

Vinzenz Wyss, Michael Schanne, Annina Stoffel: Medienkritik in der Schweiz - eine Bestandesaufnahme. SQM 4/2012. 18 Seiten. PDF E-Book. ISBN 978-3-7965-3230-6. Ca. sFr. 12.- / € (D) 12.- / € (A) 12.50.

Esther Kamber, Kurt Imhof: Nordwest- und Südostschweiz: Konzentration und publizistisches Angebot im regionalen Pressemarkt. SQM 1/2011. 20 Seiten. PDF E-Book. ISBN 978-3-7965-3222-1. Ca. sFr. 12.- / € (D) 12.- / € (A) 12.50.

Linards Udris, Kurt Imhof, Patrik Ettinger: Problematisierung des Fremden in der direkten Demokratie. SQM 2/2011. 33 Seiten. PDF E-Book. ISBN 978-3-7965-3223-8. Ca. sFr. 12.- / € (D) 12.- / € (A) 12.50.

Mark Eisenegger, Sibylle Oetiker, Mario Schranz: Monopol der Schweizerischen Depeschenagentur (sda) und Übernahmepraxis von Agenturberichten in der Presse. SQM 3/2011. 27 Seiten. PDF E-Book. ISBN 978-3-7965-3224-5. Ca. sFr. 12.- / $€(D) 12 .-/ €(A) 12.50$.

Pascal Bürgis, Angelo Gisler, Mark Eisenegger: Einfluss von Public Relations in der Unternehmensberichterstattung. SQM 4/2011. 21 Seiten. PDF E-Book. ISBN 978-3-7965-3225-2. Ca. sFr. 12.- / € (D) 12.- / € (A) 12.50.

Colin Porlezza, Stephan Russ-Mohl, Marta Zanichelli: Die doppelte Schwachstelle: Fehlerhäufigkeit und Corrections Management. SQM 5/2011. 18 Seiten. PDF E-Book. ISBN 978-3-7965-3226-9. Ca. sFr. 12.- / € (D) 12.- / € (A) 12.50.

Patrik Ettinger: Qualität der Medienberichterstattung zur Minarettinitiative. SQM 1/2010. 10 Seiten. PDF E-Book. ISBN 978-3-7965-3220-7. Open Access.

Mario Schranz, Mark Eisenegger, Kurt Imhof, Jörg Schneider: Wirtschaftsberichterstattung in der Krise. SQM 2/2010. 11 Seiten. PDF E-Book. ISBN 978-3-7965-3221-4. Open Access.

\section{Reflexionen Qualität der Medien}

\section{Schweiz - Suisse - Svizzera}

Herausgegeben vom fög - Forschungsinstitut Öffentlichkeit und Gesellschaft / Universität Zürich im Auftrag der Kurt Imhof Stiftung für Medienqualität, Zürich. ISSN 2296-8393.

Peter Studer: Leisten Presseräte, was sie sollen und wollen? Der Schweizer Presserat im Vergleich mit dem schwedischen, deutschen und britischen Modell. RQM 1/2014. 23 Seiten. PDF E-Book. ISBN 978-3-7965-3345-7. Ca. sFr. 12.- / € (D) 12.-/ $€($ A) 12.50 . 
Qualität der Medien

Schweiz - Suisse - Svizzera

Herausgegeben vom fög - Forschungsinstitut

Öffentlichkeit und Gesellschaft / Universität Zürich

im Auftrag der Stiftung Öffentlichkeit

und Gesellschaft, Zürich

\section{Jahrbuch 2016 \\ Qualität der Medien}

2016. 156 Seiten. Gebunden.

Zahlreiche Abbildungen, Grafiken und Tabellen.

Ca. sFr. 59.- / € (D) 59.-

Bei Abnahme im Abonnement:

Ca. sFr. 49.- / € (D) 49.-

ISBN 978-3-7965-3550-5

ISSN 1664-4131

E-Book: www.schwabeverlag.ch

ISBN E-Book 978-3-7965-3551-2

ISSN E-Book 2296-7931

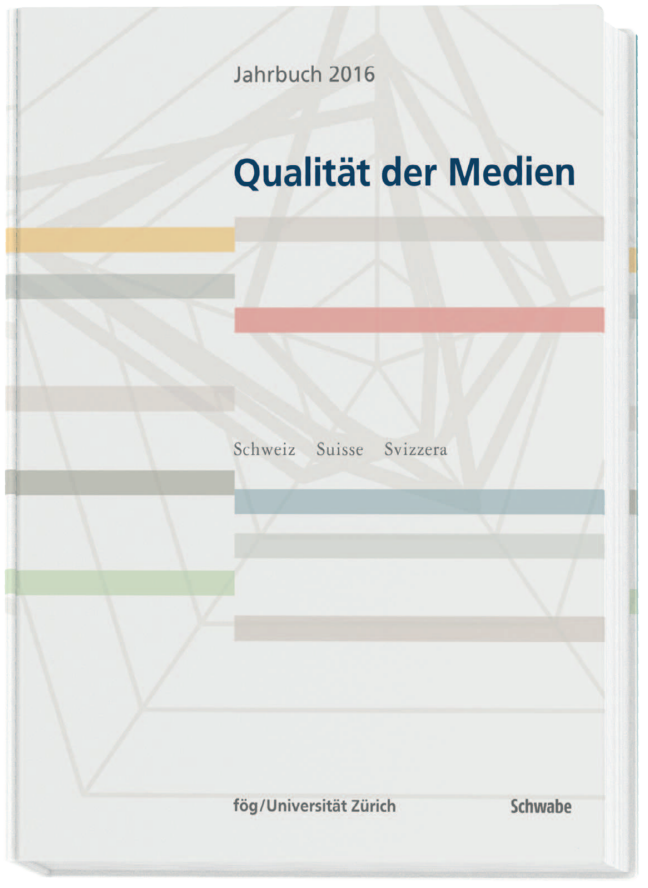

\section{Schweizer Medien unter der Lupe}

Die siebte Ausgabe Jahrbuch Qualität der Medien ist von zwei Neuerungen geprägt. Einerseits wurde die Methodik des Qualitätsscorings erheblich erweitert. Das etablierte Konzept wurde um weitere Indikatoren ergänzt, was u.a. eine noch validere Messung der Qualitätsdimension Vielfalt ermöglicht. Die Daten fliessen auch in das Forschungsprojekt Medienqualitätsrating (MQR-16) ein, das die Analyse der Berichterstattungsqualität mit einer Befragung zur Qualitätswahrnehmung des Publikums kombiniert. Mit dieser Befragung konnte empirisch bestätigt werden, dass die Nutzerinnen und Nutzer das dem Jahrbuch Qualität der Medien zugrunde liegende normative Qualitätsverständnis teilen. Andererseits ist das fög neu an einer Grossstudie beteiligt, die durch das Reuters Institute for the Study of Journalism an der University of Oxford durchgeführt wird. Der Bericht Reuters Digital News Report erscheint jährlich und enthält Umfragedaten zum Mediennutzungsverhalten für 26 Länder, neu auch für die Schweiz. Die umfassenden
Ergebnisse dieser Studie werden ab diesem Jahr in die Analysen des Jahrbuchs Qualität der Medien einbezogen. Sie ermöglichen es beispielsweise, detaillierte Erkenntnisse über die Mediennutzungspräferenzen von jungen Erwachsenen in der Schweiz und im internationalen Vergleich zu gewinnen.

Das Jahrbuch Qualität der Medien ist eine informative Quelle für Medienschaffende, Führungskräfte aus Politik und Wirtschaft, für die Wissenschaft und alle, die sich mit der Entwicklung unserer Medien und ihren Inhalten auseinandersetzen wollen. Es will das Bewusstsein für die Qualität der Medien stärken und die Diskussion über den Wandel unserer medialen Öffentlichkeit anregen.

2016 erscheinen folgende Studien:

- Medienvertrauen - eine vergleichende Perspektive

- Wie Mediennutzer in die Welt schauen: Die Newsrepertoires der Schweizerinnen und Schweizer und ihre Themenagenden
Das Jahrbuch Qualität der Medien - Schweiz Suisse Svizzera wird erarbeitet durch das fög - Forschungsinstitut Öffentlichkeit und Gesellschaft / Universität Zürich (www.foeg.uzh.ch). Es wird gefördert durch die Kurt Imhof Stiftung für Medienqualität (www.kurt-imhof-stiftung.ch). Sein Ziel ist es, das Qualitätsbewusstsein für die Medien in der Schweiz zu stärken.

\section{Studien und Reflexionen Qualität der Medien}

Neben dem Jahrbuch erscheinen auch die E-Journals Studien und Reflexionen Qualität der Medien.

Sie sind erhältlich unter: www.schwabeverlag.ch ISSN Studien 2296-5114

ISSN Reflexionen 2296-8393 